\title{
Cyclic plastic hinges with degradation effects for frame structures
}

Tidemann, Lasse; Krenk, Steen

Published in:

Journal of Engineering Mechanics - ASCE

Link to article, DOI:

10.1061/(ASCE)EM.1943-7889.0001358

Publication date:

2017

Document Version

Peer reviewed version

Link back to DTU Orbit

Citation (APA):

Tidemann, L., \& Krenk, S. (2017). Cyclic plastic hinges with degradation effects for frame structures. Journal of Engineering Mechanics - ASCE, 143(12), [04017142]. https://doi.org/10.1061/(ASCE)EM.1943-7889.0001358

\section{General rights}

Copyright and moral rights for the publications made accessible in the public portal are retained by the authors and/or other copyright owners and it is a condition of accessing publications that users recognise and abide by the legal requirements associated with these rights.

- Users may download and print one copy of any publication from the public portal for the purpose of private study or research.

- You may not further distribute the material or use it for any profit-making activity or commercial gain

- You may freely distribute the URL identifying the publication in the public portal

If you believe that this document breaches copyright please contact us providing details, and we will remove access to the work immediately and investigate your claim 


\title{
Cyclic Plastic Hinges with Degradation Effects for Frame Structures
}

\author{
Lasse Tidemann $^{1,2}$ and Steen Krenk ${ }^{1}$
}

\begin{abstract}
A model of cyclic plastic hinges in frame structures including degradation effects for stiffness and strength is developed. The model is formulated via potentials in terms of section forces. It consists of a yield surface, described in a generic format permitting representation of general convex shapes including corners, and a set of evolution equations based on an internal energy potential and a plastic flow potential. The form of these potentials is specified by five parameters for each generalized stress-strain component describing: yield level, ultimate stress capacity, elastic and elasto-plastic stiffness, and a shape parameter. The model permits gradual changes in stiffness and strength parameters via damage-based degradation. The degradation effects are introduced in the energy and flow potentials and result in additional evolution equations for the corresponding strength and stiffness parameters. The cyclic plastic hinges are introduced into a six-component equilibrium-based beam element, using additive element and hinge flexibilities. When converted to stiffness format the plastic hinges are incorporated into the element stiffness matrix. The cyclic plastic hinge model has been implemented in a computer program and used for analysis of some simple structures illustrating the characteristic features of the cyclic response and the accuracy of the proposed model.
\end{abstract}

Keywords: Cyclic plasticity; plastic hinges; frame structures; damage effects

\section{INTRODUCTION}

The concept of plastic hinges has been widely used for ultimate load carrying capacity analysis with focus on monotonically varying loads (Powell and Chen 1986; Liew et al. 1993; Attalla et al. 1994; Krenk et al. 1999), but also to a more limited extent for analysis with cyclic plasticity as e.g. earthquake response analysis, taking into account some kind of degradation effect (Inglessis et al. 1999; Kaewkulchai and Williamson 2004). The theory of plastic hinges was introduced in the late 1960's for both monotonic loading (Ueda et al. 1968), and cyclic loading with large displacements (Ueda et al. 1969). The elastic tangent stiffness matrix in a large displacement but small deformation theory was derived in (Oran 1973) with the use of an equilibrium format of the beam. The theory of concentrated yield hinges in beams was further extended to a general plastic node method (Ueda and Yao 1982) with extensions to e.g. plate elements. Further works on plastic hinge theory for beams include analysis of the effect of geometric nonlinearity (Liew et al. 2000), spread of plasticity (Jiang et al. 2002), and plastic behaviour during fire (Iu and Chan 2004).

\footnotetext{
${ }^{1}$ Department of Mechanical Engineering, Technical University of Denmark, DK-2800 Kgs. Lyngby, Denmark

${ }^{2}$ Facilities \& Projects Discipline Area, Maersk Oil A/S, DK-6700 Esbjerg, Denmark
} 
One of the early works on cyclic plasticity with degradation in relation to structures (Baber and Wen 1981), focused on random vibrations, but emphasized the importance of degradation mechanisms and the identification of these in the mathematical model as well as the separation of the individual mechanisms in the model. Later works (Ibarra et al. 2005; Lignos and Krawinkler 2011; Kamaris et al. 2013) have adopted the concept of separation of the mechanisms by modelling each section force component separately with predefined hysteresis and backbone curves and describing relevant model parameters statistically. Degradation mechanisms have also been introduced into beam elements via the flexibility format, in which plasticity and degradation have been combined using specified degradation functions, (Inglessis et al. 1999; Cipollina et al. 1995).

The degradation mechanisms typically include degradation of elastic stiffness, elastoplastic stiffness, the yield capacity of the different section force components, and the ultimate capacity of the section force components. The effects are experimentally observed in reinforced concrete structures, (Lu et al. 1999; Masi et al. 2013), where a substantial part of the degradation originates from cracking in the concrete, and in steel structures, (Popov et al. 1980; Mamaghani and Kajikawa 1998; Elchalakani et al. 2003; Elchalakani 2007), where the degradation mechanisms typically include fracture and local buckling.

Key ingredients in plasticity theories are the yield surface and the gradient of the flow potential, that may be obtained for beam cross-sections using analytical expressions (Chen and Atsuta 2008), or numerical estimates (Liu et al. 2009). However, for most practical purposes it is of interest to use an approximate representation of the yield surface and the flow potential, as most analytical yield surfaces describing beam cross-sections are based on ideal plasticity and have corners with undefined gradients, (Chen and Atsuta 2008). A multi-linear approximation of experimental yield surfaces also introduces corners, and certain measures have to be taken to overcome the problem with undefined gradients at the corners as described in e.g. (Krenk et al. 1993). Furthermore, a multi-linear representation leads to multiple algorithmic checks for violation of the yield constraint, a complication that may be circumvented by approximating the yield surface using a single-equation approximation, see e.g. (Kitipornchai et al. 1991). To overcome problems with undefined gradients at corners in a multi-linear approximation a single-equation representation of the yield function as a sum of even powers of the generalized stress components was proposed for I-beams in (Orbison et al. 1982). However, it turned out to be quite difficult to guarantee convexity of yield surfaces of this format. In contrast, convexity is ensured when using ellipsoids for approximation of the yield surface, and an early proposal (Willam and Warnke 1974) for the use of an elliptical approximation of yield surfaces has been fairly widely adopted, e.g. in (Folino et al. 2009). However, the determination of the necessary gradients may be elaborate and the format has limited flexibility with regard to general 
geometric shapes.

In order to increase the flexibility of the representation various methods known from isogeometric analysis have been proposed during recent years. A NURBS-based approach was suggested in (Coombs et al. 2016), that can in principle accurately approximate any yield surface, provided the control points can be chosen appropriately. Hardening may be introduced via movement of the control points, providing an option of modifying the shape of the yield surface locally during hardening. Depending on the number of control points it may be cumbersome though and special care has to be taken to ensure convexity of the surface. An alternative quite simple and flexible generic surface format using a Minkowski sum of ellipsoids ensuring convexity, was suggested by (Bleyer and de Buhan 2013a; Bleyer and de Buhan 2013b). The format has a high accuracy but the actual formation of the Minkowski sum as well as derivation of the gradients may be difficult.

The present paper develops a cyclic plasticity formulation for plastic hinges in beam elements. The basic evolution equations for cyclic plasticity with optional degradation of stiffness and strength is presented in Section 2, generalizing the stress based von Mises theory presented in (Krenk and Tidemann 2016) to a format in terms of normalized section forces. In Section 3 a generic yield surface format, based on the sum of square roots of quadratic forms, is introduced. This yield surface format plays a key role in representing realistic convex yield surfaces for beam cross-sections, and includes the option of smoothing sharp corners of the surface. In Section 4 the cyclic plasticity model is implemented in the form of plastic hinges into a beam element via the flexibility format from (Krenk et al. 1999), including the derivation of the consistent algorithmic tangent stiffness matrix. Finally, examples illustrate the characteristic properties of the cyclic response and the accuracy of the proposed model.

\section{PLASTIC HINGE MODEL}

A plastic hinge in a beam is a local deformation mechanism located at a cross-section of the beam in which local elongation and angle discontinuities are considered as generalized plastic strains corresponding to generalized stresses defined in terms of the local section forces. For beams the generalized stresses governing the behaviour of plastic hinges will typically be the normal force and two bending moments as shown in Fig. 1. Other section forces may also be included but are typically of minor influence.

Thus, the generalized stresses and strains used are defined as

$$
\boldsymbol{\tau}=\left[N, M_{y}, M_{z}, \ldots\right]^{T} \quad, \quad \gamma=\left[\varepsilon_{x}, \kappa_{y}, \kappa_{z}, \ldots\right]^{T} .
$$

These generalized stresses and strains are now used to define a cyclic plasticity model, 


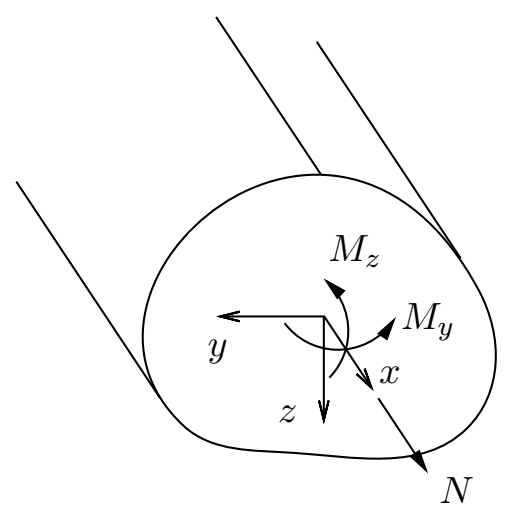

FIG. 1. Beam cross-section with section forces as generalized stresses.

following the procedure developed in (Krenk and Tidemann 2016). The first step is to introduce an internal energy function consisting of a sum of three contributions: a quadratic function of the generalized elastic strains $\gamma_{\mathrm{e}}$, a quadratic function of a correspondig set of generalized internal strains $\gamma_{i}$, and an additive function of a set of generalized strains $\boldsymbol{\xi}$ representing damage. The internal energy hereby takes the form

$$
\varphi\left(\boldsymbol{\gamma}_{\mathrm{e}}, \boldsymbol{\gamma}_{\mathrm{i}}, \boldsymbol{\xi}\right)=\frac{1}{2} \boldsymbol{\gamma}_{\mathrm{e}}^{T} \mathbb{D}_{\mathrm{e}}(\boldsymbol{\xi}) \boldsymbol{\gamma}_{\mathrm{e}}+\frac{1}{2} \boldsymbol{\gamma}_{\mathrm{i}}^{T} \mathbb{D}_{\mathrm{i}}(\boldsymbol{\xi}) \boldsymbol{\gamma}_{\mathrm{i}}+\varphi_{\mathrm{d}}(\boldsymbol{\xi})
$$

The matrices $\mathbb{D}_{\mathrm{e}}(\boldsymbol{\xi})$ and $\mathbb{D}_{\mathrm{i}}(\boldsymbol{\xi})$ representing the contributions from the elastic and the internal strains may depend on the damage parameters $\boldsymbol{\xi}$, thereby permitting changes in stiffness due to degradation. The energy conjugate generalized stresses are found via differentiation of the energy potential as

$$
\begin{aligned}
& \boldsymbol{\tau}=\partial_{\gamma_{\mathrm{e}}} \varphi=\mathbb{D}_{\mathrm{e}}(\boldsymbol{\xi}) \boldsymbol{\gamma}_{\mathrm{e}} \\
& \boldsymbol{\tau}_{\mathrm{i}}=\partial_{\gamma_{\mathrm{i}}} \varphi=\mathbb{D}_{\mathrm{i}}(\boldsymbol{\xi}) \gamma_{\mathrm{i}} \\
& \boldsymbol{\eta}=\partial_{\boldsymbol{\xi}} \varphi
\end{aligned}
$$

It is observed that the generalized stresses $\boldsymbol{\tau}$ and the generalized internal stresses $\boldsymbol{\tau}_{\mathrm{i}}$ defined via (3) and (4), respectively, have a Hooke's law type relation to their energy conjugate generalized strains.

\section{Normalized yield surface and flow potential}

In plasticity models like the present, based on generalized stresses representing different quantities like forces and moments, plasticity parameters like yield and ultimate stress levels may be different and also may develop differently during plastic loading. This suggests the use of the normalized section forces $\left[n, m_{y}, m_{z}, \ldots\right]=\left[N / N^{y}, M_{y} / M_{y}^{y}, M_{z} / M_{z}^{y}, \ldots\right]$ in the formulation of the yield surface and the flow potential. This corresponds to using normalized generalized stresses $\tilde{\boldsymbol{\tau}}$ and normalized internal stresses $\tilde{\boldsymbol{\tau}}_{\mathrm{i}}$ defined by

$$
\tilde{\boldsymbol{\tau}}(\boldsymbol{\eta})=\mathbb{B}_{y}^{-1} \boldsymbol{\tau} \quad, \quad \tilde{\boldsymbol{\tau}}_{\mathrm{i}}(\boldsymbol{\eta})=\mathbb{B}_{y}^{-1} \boldsymbol{\tau}_{\mathrm{i}}
$$


where the normalization coefficients are arranged in the diagonal matrix

$$
\mathbb{B}_{y}(\boldsymbol{\eta})=\left[\begin{array}{cccc}
N^{y}(\boldsymbol{\eta}) & & & \\
& M_{y}^{y}(\boldsymbol{\eta}) & & \\
& & M_{z}^{y}(\boldsymbol{\eta}) & \\
& & & \ddots
\end{array}\right] .
$$

It is convenient to choose the normalization coefficients as the current yield level for the corresponding single component load.

In cyclic plasticity the yield surface moves in stress space, and the simplest format consists of a translation of the yield surface described by the generalized internal stresses $\boldsymbol{\tau}_{\mathrm{i}}$, the so-called kinematic hardening. This is conveniently represented by the generic yield function format

$$
F\left(\boldsymbol{\tau}, \boldsymbol{\tau}_{\mathrm{i}}, \boldsymbol{\eta}\right)=\left\|\tilde{\boldsymbol{\tau}}(\boldsymbol{\eta})-\tilde{\boldsymbol{\tau}}_{\mathrm{i}}(\boldsymbol{\eta})\right\|-1
$$

where \|\| is a suitable norm of the generalized stresses. It is well known from von Mises plasticity that a particularly simple formulation is obtained when the yield condition is expressed via the equivalent stress $\sigma_{\mathrm{e}}$, which is a homogeneous form of degree one in the stress components. This concept is extended to a more general yield function of degree one in Section 3.

The basic form of the flow potential is constructed in a way similar to the flow potential in the stress-based model in (Krenk and Tidemann 2016), where a quadratic term of the normalized internal generalized stresses $\tilde{\boldsymbol{\tau}}_{\mathrm{i}}$ is added to the yield function along with an unspecified function of the stress-like damage parameters, $\boldsymbol{\eta}$, giving the flow potential

$$
G\left(\boldsymbol{\tau}, \boldsymbol{\tau}_{\mathrm{i}}, \boldsymbol{\eta}\right)=F\left(\boldsymbol{\tau}, \boldsymbol{\tau}_{\mathrm{i}}, \boldsymbol{\eta}\right)+\frac{1}{2} \tilde{\boldsymbol{\tau}}_{\mathrm{i}}^{T} \mathbb{B}_{\beta}^{-1} \tilde{\boldsymbol{\tau}}_{\mathrm{i}}+G_{\mathrm{d}}(\boldsymbol{\eta})
$$

where $\tilde{\boldsymbol{\tau}}_{\mathrm{i}}=\tilde{\boldsymbol{\tau}}_{\mathrm{i}}(\boldsymbol{\eta})$ and the coefficient matrix $\mathbb{B}_{\beta}$ is defined as

$$
\mathbb{B}_{\beta}(\boldsymbol{\eta})=\left[\begin{array}{cccc}
\beta_{N}(\boldsymbol{\eta}) & & & \\
& \beta_{M_{y}}(\boldsymbol{\eta}) & & \\
& & \beta_{M_{z}}(\boldsymbol{\eta}) & \\
& & & \ddots
\end{array}\right] .
$$

In the yielding process the yield surface moves as described by the normalized center stress $\tilde{\boldsymbol{\tau}}_{\mathrm{i}}$. The first term in the flow potential (9) is of degree one, while the second term is of degree two. Hereby the parameters in the array $\boldsymbol{\beta}=\left[\beta_{N}, \beta_{M_{y}}, \beta_{M_{z}}, \ldots\right]^{T}$ determine the limits on the normalized center stress components $\tilde{\tau}_{\mathrm{i}}$, thereby defining the ultimate stress capacity. In the von Mises stress-based model, (Krenk and Tidemann 2016), the ratio between ultimate and initial yield level, is given by a single parameter corresponding to $1+\beta$, while in the present model the additional capacity is defined individually for each generalized stress component. 


\section{Generalized stress evolution matrix}

The evolution equations for external stresses, internal stresses and damage parameters are derived from the assumption of maximum dissipation rate. The dissipation rate is expressed as the rate of externally supplied energy $\boldsymbol{\tau} \dot{\gamma}$ minus the change in the internal energy represented by $\dot{\varphi}$,

$$
\dot{\mathcal{D}}=\boldsymbol{\tau} \dot{\gamma}-\dot{\varphi}\left(\gamma_{\mathrm{e}}, \gamma_{\mathrm{i}}, \boldsymbol{\xi}\right) \geq 0
$$

It is assumed that the observable generalized strain $\gamma$ is the sum of the generalized elastic strain $\gamma_{\mathrm{e}}$ and the generalized plastic strain $\gamma_{\mathrm{p}}$. The evolution equations for the generalized stresses and damage parameters are derived by maximizing the dissipation rate, under the constraint that the material can be described by the flow potential, $G\left(\boldsymbol{\tau}, \boldsymbol{\tau}_{\mathrm{i}}, \boldsymbol{\eta}\right)$, and the consistency condition $\dot{F}\left(\boldsymbol{\tau}, \boldsymbol{\tau}_{\mathrm{i}}, \boldsymbol{\eta}\right)=0$. A more detailed derivation is given in (Krenk and Tidemann 2016). The elasto-plastic stiffness matrix is represented in a very compact form by introduction of the combined external, internal and damage evolution matrix

$$
\begin{aligned}
& \mathbb{D}_{\mathrm{eid}}(\boldsymbol{\xi})=\left[\begin{array}{ccc}
\mathbb{D}_{\mathrm{e}}(\boldsymbol{\xi}) & \mathbf{0} & \partial_{\boldsymbol{\xi}}^{T} \boldsymbol{\tau} \\
\mathbf{0} & \mathbb{D}_{\mathrm{i}}(\boldsymbol{\xi}) & \partial_{\boldsymbol{\xi}}^{T} \boldsymbol{\tau}_{\mathrm{i}} \\
\left(\partial_{\boldsymbol{\xi}}^{T} \boldsymbol{\tau}\right)^{T} & \left(\partial_{\boldsymbol{\xi}}^{T} \boldsymbol{\tau}_{\mathrm{i}}\right)^{T} & \partial_{\boldsymbol{\xi}}^{T} \boldsymbol{\eta}
\end{array}\right], \\
& \mathbb{D}_{\mathrm{ed}}(\boldsymbol{\xi})=\left[\begin{array}{lll}
\mathbb{D}_{\mathrm{e}}(\boldsymbol{\xi}) & \mathbf{0} & \partial_{\boldsymbol{\xi}}^{T} \boldsymbol{\tau}
\end{array}\right]^{T},
\end{aligned}
$$

where $\mathbb{D}_{\text {ed }}$ represents the stiffness relating an increment in the generalized external strains $\dot{\gamma}_{\mathrm{e}}$ to increments in the generalized external stresses $\dot{\tau}$, internal stresses $\dot{\tau}_{\mathrm{i}}$ and damage stresses, $\dot{\boldsymbol{\eta}}$. The matrix $\mathbb{D}_{\text {ed }}$ corresponds to the first column of $\mathbb{D}_{\text {eid }}$ in the definition (12).

The gradients of the yield surface and the flow potential are a key part of the determination of the elasto-plastic stiffness matrix, and these are defined in vector form as

$$
\begin{aligned}
& \boldsymbol{\partial} F=\left[\partial_{\boldsymbol{\tau}}^{T} F, \partial_{\boldsymbol{\tau}_{\mathrm{i}}}^{T} F, \partial_{\boldsymbol{\eta}}^{T} F\right]^{T}, \\
& \boldsymbol{\partial} G=\left[\partial_{\boldsymbol{\tau}}^{T} G, \partial_{\boldsymbol{\tau}_{\mathrm{i}}}^{T} G, \partial_{\boldsymbol{\eta}}^{T} G\right]^{T}
\end{aligned}
$$

Hereby the evolution equations of the generalized stresses and stress-like parameters take the form

$$
\left[\begin{array}{c}
\dot{\boldsymbol{\tau}} \\
\dot{\boldsymbol{\tau}}_{\mathrm{i}} \\
\dot{\boldsymbol{\eta}}
\end{array}\right]=\left[\mathbb{D}_{\mathrm{ed}}-\frac{\mathbb{D}_{\text {eid }}(\boldsymbol{\partial} G)(\boldsymbol{\partial} F)^{T} \mathbb{D}_{\mathrm{ed}}}{(\boldsymbol{\partial} F)^{T} \mathbb{D}_{\text {eid }}(\boldsymbol{\partial} G)}\right] \dot{\boldsymbol{\gamma}}
$$

From (16) the elasto-plastic stiffness is identified as

$$
\mathbb{D}_{\text {eid }}^{\text {ep }}=\mathbb{D}_{\text {ed }}-\frac{\mathbb{D}_{\text {eid }}(\boldsymbol{\partial} G)(\boldsymbol{\partial} F)^{T} \mathbb{D}_{\text {ed }}}{(\boldsymbol{\partial} F)^{T} \mathbb{D}_{\text {eid }}(\boldsymbol{\partial} G)}
$$


where the subscript 'eid' indicates that the elasto-plastic stiffness matrix relates to the generalized external, internal and damage stresses, and the superscript 'ep' indicates the elasto-plastic stiffness matrix. With this formulation $\mathbb{D}_{\mathrm{e}}$ describes the elastic stiffness, $\mathbb{D}_{\mathrm{i}}$ describes the tangent stiffness at initial yield, and the $\beta$-parameters describe the relative value of the ultimate capacity of the respective generalized stress components.

\section{Plastic straining and enhanced flow potential}

The elastic stiffness is described by the stiffness matrix $\mathbb{D}_{\mathrm{e}}$. At initial yield additive plastic strains appear, and if disregarding possible damage effects in this particular context, the initial elasto-plastic stress-strain relation follows from (16) in the form

$$
\dot{\boldsymbol{\tau}}=\mathbb{D}_{\mathrm{e}} \dot{\gamma}-\mathbb{D}_{\mathrm{e}}\left(\partial_{\tau} G\right) \frac{\left(\partial_{\tau}^{T} F\right) \mathbb{D}_{\mathrm{e}} \dot{\gamma}}{\left(\partial_{\tau}^{T} F\right) \mathbb{D}_{\mathrm{e}}\left(\partial_{\tau} G\right)+\left(\partial_{\tau_{\mathrm{i}}}^{T} F\right) \mathbb{D}_{\mathrm{i}}\left(\partial_{\tau_{\mathrm{i}}} G\right)}
$$

In general, the elastic and the plastic strain rates may have different direction. However, if assuming that the directions are identical, amounting to proportionality between the strain rate and the stress gradient of the flow potential, $\dot{\gamma} \propto \partial_{\tau} G$, the stress-strain evolution relation (18) can be expressed in the form

$$
\dot{\tau}=\frac{\mathbb{D}_{\mathrm{e}} \dot{\gamma}}{1+\frac{\left(\partial_{\tau}^{T} F\right) \mathbb{D}_{\mathrm{e}}\left(\partial_{\tau} G\right)}{\left(\partial_{\tau_{\mathrm{i}}}^{T} F\right) \mathbb{D}_{\mathrm{i}}\left(\partial_{\tau_{\mathrm{i}}} G\right)}}
$$

In this formula the second term in the denominator represents the relative increase in flexibility due to the additional plastic straining. It is seen that this term depends on the ratio of the internal stiffness $\mathbb{D}_{\mathrm{i}}$ to the external stiffness $\mathbb{D}_{\mathrm{e}}$. The effect of the relative magnitude of the internal stiffness is illustrated in Fig. 2(a), in which the relative magnitude of the internal stiffness is 2.0, 1.0 and 0.5, respectively. It is seen that for a large value of the relative internal stiffness the kink in the stress-strain curve at beginning yield becomes small, and conversely.

While the initial inclination of the stress-strain relation at initial yield is governed by the relative magnitude of the internal stiffness parameters, the subsequent development of plastic straining, and thereby the generalized stress-strain relation, between initial yield and the ultimate capacity is governed by the gradient of the internal stress term in the flow potential. With the flow potential of the basic form indicated in (9) the gradient with respect to the (normalized) internal stress components is given by the partial derivatives,

$$
\partial_{\tilde{\tau}_{j}^{\mathrm{i}}} G=\partial_{\tilde{\tau}_{j}^{\mathrm{i}}} F+\frac{\tilde{\tau}_{j}^{\mathrm{i}}}{\beta_{j}} .
$$

This form leads to a development of the plastic straining determined by the yield and ultimate stress levels and the initial slope at first yield. As demonstrated in (Krenk 
(a)

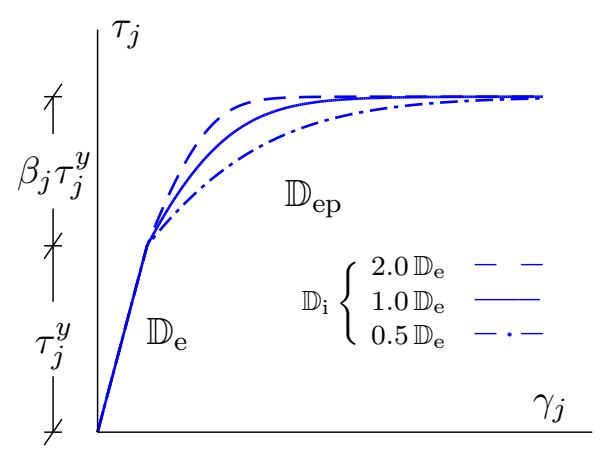

(b)

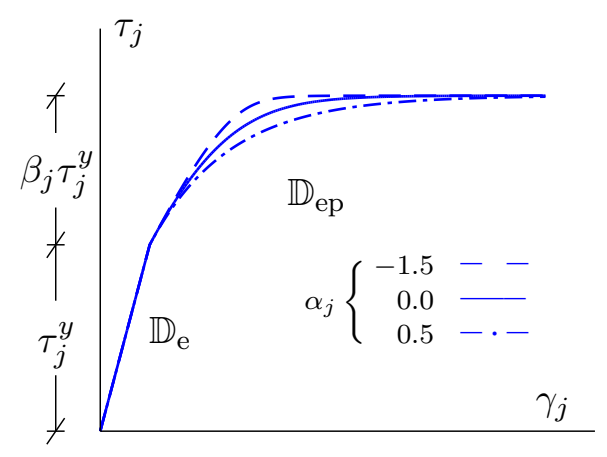

FIG. 2. Influence of parameters on stress-strain relation: (a) relative internal stiffness $\mathbb{D}_{\mathrm{i}}=(-) \mathbb{D}_{\mathrm{e}}$, (b) modification of flow potential $G$ via the shape parameter $\alpha_{j}$.

and Tidemann 2016) for the von Mises stress-based model, it is possible to enhance the flow potential, making it possible to control the development of plastic straining between the point of initial yield and ultimate stress level. In the present context the hysteresis curves for the individual generalized stress components may be different, and thus must be defined by parameters each associated with a specific generalized stress component. A quite flexible format is obtained by replacing the denominator $\beta_{j}$ by a weighted average of the parameter $\beta_{j}$ and the current absolute value of the corresponding normalized internal stress component $\tilde{\tau}_{j}^{\mathrm{i}}$. Hereby the components of the flow potential gradient take the form

$$
\partial_{\tilde{\tau}_{j}^{\mathrm{i}}} G=\partial_{\tilde{\tau}_{j}^{\mathrm{i}}} F+\frac{\tilde{\tau}_{j}^{\mathrm{i}}}{\left(1-\alpha_{j}\right) \beta_{j}+\alpha_{j}\left|\tilde{\tau}_{j}^{\mathrm{i}}\right|},
$$

where $\alpha_{j}$ is the weight parameter for component $j$. This formulation enables different $\alpha$ parameters for each generalized stress component. Integration of the gradient components defined in (21) leads to the following form of the flow potential,

$$
\begin{aligned}
G\left(\boldsymbol{\tau}, \boldsymbol{\tau}_{\mathrm{i}}, \boldsymbol{\eta}\right)= & F\left(\boldsymbol{\tau}, \boldsymbol{\tau}_{\mathrm{i}}, \boldsymbol{\eta}\right)+G_{\mathrm{d}}(\boldsymbol{\eta}) \\
& +\sum_{j} \frac{1}{\alpha_{j}}\left\{\left|\tilde{\tau}_{j}^{\mathrm{i}}\right|-\frac{1-\alpha_{j}}{\alpha_{j}} \beta_{j} \ln \left(1+\frac{\alpha_{j}}{1-\alpha_{j}} \frac{\left|\tilde{\tau}_{j}^{\mathrm{i}}\right|}{\beta_{j}}\right)\right\},
\end{aligned}
$$

where $\alpha_{j}=\alpha_{j}(\boldsymbol{\eta}), \beta_{j}=\beta_{j}(\boldsymbol{\eta})$ and $\tilde{\tau}_{j}^{\mathrm{i}}=\tilde{\tau}_{j}^{\mathrm{i}}(\boldsymbol{\eta})$. The basic potential from (9) corresponds to the limit $\alpha_{j}=0$, while as demonstrated in (Krenk and Tidemann 2016) more representative values for von Mises plasticity were found to be in the order of $\alpha_{j}=0.7-0.9$. The effect of the shape parameter $\alpha_{j}$ is illustrated in Fig. 2(b) showing stress-strain curves for $\alpha_{j}=-1.5,0.0$ and 0.5. Appropriate combination of the parameter(s) $\alpha_{j}$ and the relative magnitude of the internal stiffness gives a very versatile representation of the shape of the elastic-plastic stress-strain curve with only two parameters per generalized stress component. The representation of the cyclic behavior is illustrated and discussed in connection with specific examples in Section 5. 


\section{GENERIC YIELD SURFACE}

The gradients of the yield surface and the flow potential are of central importance, as they determine the basic properties of the elasto-plastic evolution matrix (16), as well as the algorithmic properties used in return algorithms in the numerical computations. In the case of materials that can be represented by the von Mises yield criterion the gradients are well-defined over the entire yield surface, whereas that may not be the case for yield surfaces representing beam cross-sections, as these may typically have corners. This problem can be overcome in various ways, e.g. with use of a locally modified yield function for tubular beam cross-sections, (Krenk et al. 1999). However, most techniques of that type require individual extensions for different types of cross-sections and hence a study of a proper extension of the yield function is necessary for each type of cross-section. Additionally, return algorithms and consistent tangent operators have to be derived for each individual yield surface, an undesirable feature in a space frame program where multiple different beam cross-sections may be needed. In order to circumvent the corner problem and the need for a library of different yield function formats a generic yield surface representation is proposed in the following.

\section{A simple approach}

The simplest form of a guaranteed convex representation, excluding the degenerate case of straight lines or planes, is an ellipsoid (in 2D an ellipse but the term ellipsoid will be used about the general $n$-dimensional version in the following). In (Skordeli and Bisbos 2010) the use of approximating yield surfaces for frame structures in the form of a single ellipsoid was suggested. However, this approximation is not very flexible, and degenerates to a sphere when expressed in terms of the normalized generalized stresses. There are two logical possibilities for a next step in the line of simple suggestions: make use of a 'super ellipsoid', where the exponent is larger than 2; or make use of the convexity guaranteed by the mathematical representation of an ellipsoid and add several of these together, leading to a convex surface. The latter approach is the more general and is chosen here.

A single ellipsoid can be represented as a homogeneous form of degree one

$$
\sqrt{\mathbf{x}^{\prime T} \mathbf{A x}}=1
$$

when $\mathbf{A}$ is a symmetric, positive definite matrix and $\mathbf{x}^{\prime}$ are the local coordinates of the ellipsoid, which may be translated and rotated relative to the global coordinate system, $\mathbf{x}$. The homogeneous form of degree one is desirable as the von Mises yield function with equivalent stress $\sigma_{\mathrm{e}}$ is homogeneous of degree one, suggesting that an ellipsoidal representation in the form (23) qualitatively has the same properties as the von Mises yield surface. An addition of terms of the type (23) with different matrices $\mathbf{A}_{1}, \mathbf{A}_{2}$, 
.. does not reduce to an ellipsoid, even if the centres are the same, and thus a format represented as a sum of terms of the type (23) offers the possibility of representing more general shapes. The suggested format is therefore

$$
\sqrt{\mathbf{x}_{1}^{\prime T} \mathbf{A}_{1} \mathbf{x}_{1}^{\prime}}+\sqrt{{\mathbf{x}_{2}^{\prime T}}^{\mathbf{A}_{2} \mathbf{x}_{2}^{\prime}}}+\cdots=1
$$

which is guaranteed convex because of the convexity of each of the terms, and where (23) represents a single ellipsoid, the sum (24) represents a combination of ellipsoids. The ratio of the axes of an ellipsoid is given by the matrix $\mathbf{A}$ whereas the finite size of the ellipsoid is given by the right-hand side in (23). As the combination of ellipsoidal terms (24) does not identify the finite size of the individual terms an infinity of combinations of finite sized ellipsoids exist. Assigning each ellipsoid a finite size, i.e. equalling each ellipsoidal term to a value less than 1 , the intersection of the individual ellipsoids - being points in $2 \mathrm{D}$ and curves in $3 \mathrm{D}$ - will be points on the resulting surface described by (24). The concept is illustrated in Fig. 3 in a format with two ellipsoidal terms, where different combinations of finite sized ellipsoids result in a convex but non-ellipsoidal surface.

(a)

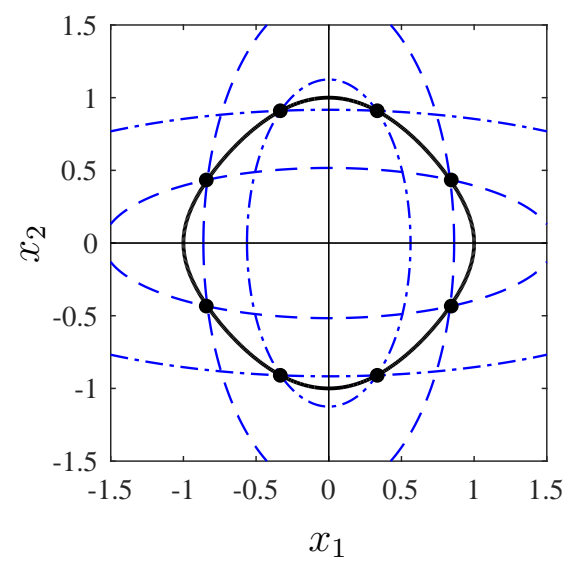

(b)

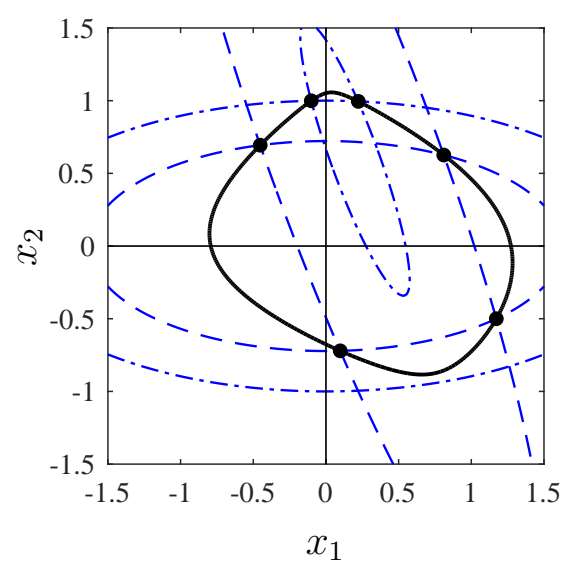

FIG. 3. Intersections of finite ellipsoids, $(-\cdot-)$ and $(--)$, are points, $(\bullet)$, on the resulting surface, (-).

Figure 3 illustrates that the suggested format can approximate both symmetric surfaces, Fig. 3(a), and non-symmetric surfaces, Fig. 3(b). Symmetric yield surfaces are typical for symmetric beam cross-sections, while non-symmetric yield surfaces are typical for non-symmetrical beam cross-sections or beams of materials with different yield strengths in tension and compression as e.g. concrete. It is furthermore observed from Fig. 3 that there is a high degree of flexibility in the approximation despite only using two terms. Including more terms than two will naturally lead to a higher degree of flexibility, but also a higher degree of complexity when determining the ratios of the semi-principal 
axes of the ellipsoids and the rotation of each ellipsoid. Because of the high degree of flexibility, the ensured convexity and the elimination of corners (the gradient of each ellipsoidal term is defined everywhere) the following generic yield surface format is suggested

$$
F\left(\boldsymbol{\tau}, \boldsymbol{\tau}_{\mathrm{i}}, \boldsymbol{\eta}\right)=\sqrt{\overline{\boldsymbol{\tau}}_{1}^{T} \mathbf{A}_{1} \overline{\boldsymbol{\tau}}_{1}}+\sqrt{\overline{\boldsymbol{\tau}}_{2}^{T} \mathbf{A}_{2} \overline{\boldsymbol{\tau}}_{2}}+\cdots-1
$$

where $\mathbf{A}_{k}$ is a positive definite, symmetric matrix for $k=1,2, \cdots$ and

$$
\overline{\boldsymbol{\tau}}_{k}(\boldsymbol{\eta})=\tilde{\boldsymbol{\tau}}(\boldsymbol{\eta})-\tilde{\boldsymbol{\tau}}_{\mathrm{i}}(\boldsymbol{\eta})-\hat{\boldsymbol{\tau}}_{k}
$$

Here $\hat{\boldsymbol{\tau}}_{k}$ is a constant offset in the normalized stress space. The number of terms as well as including offset are both options to increase the accuracy of the representation. However, for most symmetric beam cross-sections it is sufficient with two or three terms where the matrices $\mathbf{A}_{k}$ are diagonal matrices and $\hat{\boldsymbol{\tau}}_{k}=\mathbf{0}$, i.e. the ellipsoids are centered at origo and are not rotated. For reinforced concrete cross-sections though it is typically advantageous to include an offset that is the same for all terms and only offsets the yield surface on the axis of the normal force.

\section{Choosing parameters for simple yield surfaces}

Yield surfaces for typical steel beam cross-sections are symmetric and centered around origo with contour lines in the $\left(n, m_{y}, 0\right),\left(n, 0, m_{z}\right)$ and $\left(0, m_{y}, m_{z}\right)$-planes resembling shapes that are interpolations between a rhombus and a circle. Such shapes can be represented rather accurately with the suggested format using two ellipsoidal terms with diagonal matrices, $\mathbf{A}_{1}$ and $\mathbf{A}_{2}$, and with zero offsets, $\hat{\boldsymbol{\tau}}_{1}=\hat{\boldsymbol{\tau}}_{2}=\mathbf{0}$. Generating a proper representation of a simple, symmetric yield surface with the format (25) is most often eased by prescribing the matrix $\mathbf{A}_{1}$ and defining $\mathbf{A}_{2}$ by the intersection of the yield surface and the $n$-, $m_{y^{-}}$and $m_{z^{-}}$axes denoted by $n^{0}, m_{y}^{0}$ and $m_{z}^{0}$ respectively. The diagonal matrix $\mathbf{A}_{2}$ is defined via the constraints

$$
\sqrt{A_{1,1}^{1}}+\sqrt{A_{1,1}^{2}}=\frac{1}{\left|n^{0}\right|}, \quad \sqrt{A_{2,2}^{1}}+\sqrt{A_{2,2}^{2}}=\frac{1}{\left|m_{y}^{0}\right|}, \quad \sqrt{A_{3,3}^{1}}+\sqrt{A_{3,3}^{2}}=\frac{1}{\left|m_{z}^{0}\right|} .
$$

Typically, the values of $n^{0}=m_{y}^{0}=m_{z}^{0}=1$ in order to permit full yield capacity of each section force, but values may be subject to change in order to change geometric properties of the yield surface representation.

The shape of the yield surface is governed by the ratios of $A_{1,1}^{1}, A_{1,1}^{2}, \cdots$, leading to a fairly simple method of choosing the values of $\mathbf{A}_{1}$. In the $\left(n, m_{y}, 0\right),\left(n, 0, m_{z}\right)$ and $\left(0, m_{y}, m_{z}\right)$-planes the shape is governed by two types of ratios with different effects; ratios of type $A_{1,1}^{k} / A_{2,2}^{k}$ and ratios of type $A_{1,1}^{1} / A_{1,1}^{2}$. The first ratio type is most important, as it controls whether the shape between the intersections of the yield surface and the axes is more rhombic or circular. If the ratio is close to one, the shape will be circular whereas 
a ratio very different from one will be rhombic. The second type of ratio controls how rounded the yield surface is close to the intersections with the axes. In the $\left(n, m_{y}, 0\right)$-plane the shape around the intersection with the $m_{y}$-axis will be rounded compared to the shape around the intersection with the $n$-axis when $A_{1,1}^{1} / A_{1,1}^{2} \gg A_{2,2}^{1} / A_{2,2}^{2}$ and vice versa when $A_{1,1}^{1} / A_{2,2}^{1}>1$, i.e. for a very rhombic shape of the yield surface, there will be a corner at the intersection with the $n$-axis when $A_{1,1}^{1} / A_{1,1}^{2} \gg A_{2,2}^{1} / A_{2,2}^{2}$. With this knowledge it is fairly easy to choose the parameters $\mathbf{A}_{1}, n^{0}, m_{y}^{0}$ and $m_{z}^{0}$ to get a proper representation of a simple, symmetric yield surface. For non-symmetric yield surfaces the process of choosing the number of terms to include in (25) may be more difficult and could potentially combine with optimization routines as in (Bleyer and de Buhan 2013b).

\section{Tubular beam yield surface}

In order to illustrate the suggested method, a tubular steel cross-section is investigated. The yield function for the axial stress component of the tubular cross-section is

$$
F\left(n, m_{y}, m_{z}\right)=\sqrt{m_{y}^{2}+m_{z}^{2}}-\cos \left(\frac{\pi}{2} n\right) .
$$

At yield the function equals zero, which defines the yield surface. The yield surface is symmetric and fairly simple as illustrated in Fig. 4, and thus it is assumed that it can be accurately approximated using (25) with two terms, diagonal matrices, and without offsets, i.e. $\hat{\boldsymbol{\tau}}_{1}=\hat{\boldsymbol{\tau}}_{2}=\mathbf{0}$, based on the above discussion. The constraints (27) are applied, effectively reducing the problem to the determination of the three diagonal terms in $\mathbf{A}_{1}$, using the normalization $\left|n^{0}\right|=\left|m_{y}^{0}\right|=\left|m_{z}^{0}\right|=1$. As the yield surface (28) in the (0, $\left.m_{y}, m_{z}\right)$ plane is a circle it is chosen that $A_{2,2}^{1}=A_{3,3}^{1}$ which gives a perfect circular representation. In the two remaining planes the yield surface (28) is very rhombic and with a sharp corner at the intersection with the $n$-axis suggesting that $A_{1,1}^{1} / A_{2,2}^{1} \neq 1, A_{1,1}^{2} / A_{2,2}^{2} \neq 1$ and $A_{1,1}^{1} / A_{1,1}^{2} \gg A_{2,2}^{1} / A_{2,2}^{2}$ to get a good representation of (28) with the format (25). Therefore $\mathbf{A}_{1}$ is chosen to have the diagonal terms $A_{1,1}^{1}=1-2 \cdot 10^{-5}$ and $A_{2,2}^{1}=A_{3,3}^{1}=0.16$ and $\mathbf{A}_{2}$ given by the constraints (27) has the diagonal terms $A_{1,1}^{2}=1 \cdot 10^{-10}$ and $A_{2,2}^{2}=A_{3,3}^{2}=0.36$ whereby $A_{1,1}^{1} / A_{2,2}^{1}=6.25 \gg 1, A_{1,1}^{2} / A_{2,2}^{2}=2.8 \cdot 10^{-10} \ll 1$ and $A_{1,1}^{1} / A_{1,1}^{2}=10^{10}$ which is far bigger than $A_{2,2}^{1} / A_{2,2}^{2}=0.44$. The resulting approximation is shown in Fig. 4.

As observed from Fig. 4 the approximation is nearly indistinguishable from the original analytically determined surface. However, there are slight differences because the approximation is a smooth surface with a unique gradient everywhere, whereas the theoretical surface has singularities at the points $\left(n, m_{y}, m_{z}\right)=( \pm 1,0,0)$. Thus, for this particular yield surface it is quite easy to determine an accurate representation of the yield surface within the proposed generic yield surface format (25). 


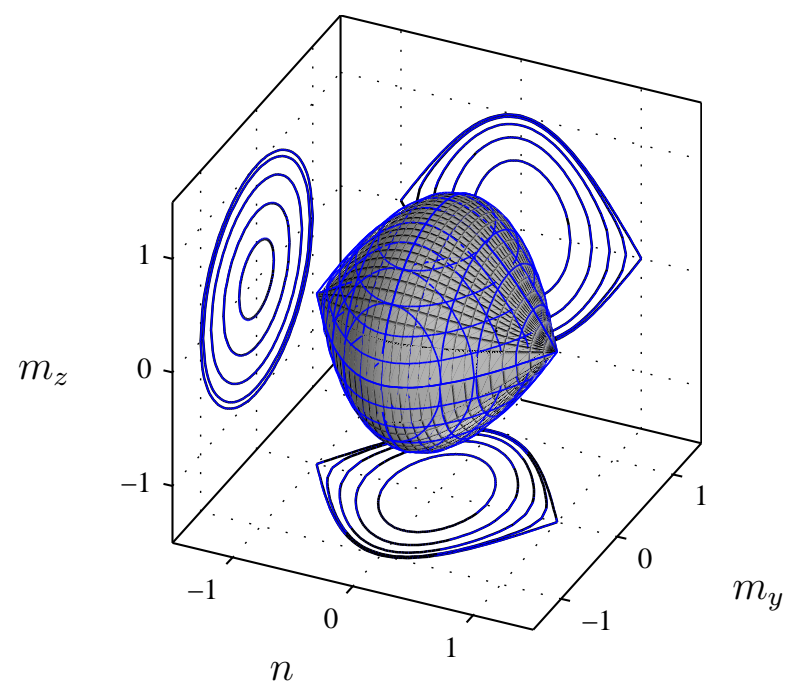

FIG. 4. Theoretical yield surface for tubular steel beam and approximation, (-). Contour lines are plotted in various planes.

\section{Exterior surfaces}

One issue that arises with the very accurate approximation is that even though the gradient is defined, where the theoretical yield surface has corners, the yield surface and the exterior surfaces still have a very large curvature in that region. The exterior surfaces are used for the return to the yield surface and especially for large load steps the return may be difficult in regions with high curvature. Due to the mathematical formulation of the yield surface (25) the exterior surfaces will simply be scaled versions of the yield surface, whereby the curvature of the exterior surfaces will be similar to that of the yield surface. Hence it may be advantageous to adjust the local curvature to facilitate the ensuing iterations. An example of how this is achieved is shown in Fig. 5 where the parameters are $\left|n^{0}\right|=0.95$, $\left|m_{y}^{0}\right|=\left|m_{z}^{0}\right|=1, A_{1,1}^{1}=0.865, A_{2,2}^{1}=A_{3,3}^{1}=0.0961$ and $\mathbf{A}_{2}$ is a diagonal matrix given by the constraints (27). The parameters are chosen such that the shape of the yield surface in the $\left(n, m_{y}, 0\right)$ - and $\left(n, 0, m_{z}\right)$-plane is still rather rhombic, suggesting $A_{1,1}^{1} / A_{2,2}^{1} \neq 1$, $A_{1,1}^{2} / A_{2,2}^{2} \neq 1$ but the shape at the intersection with the $n$-axis should be more rounded than in the accurate representation, but still somewhat resembling corners, suggesting $A_{1,1}^{1} / A_{1,1}^{2} \gg A_{2,2}^{1} / A_{2,2}^{2}$ but with a smaller factor compared to the accurate representation.

It is observed from Fig. 5 that although the slight change in the parameters does not change the representation of the analytical yield surface much, the curvature is reduced dramatically in the critical regions, thereby improving the iteration convergence rate. The fact that the generic yield surface is guaranteed convex without singularities, has gradients that are easy to determine, has a high degree of flexibility and accuracy, and can create exterior surfaces with relatively low curvature indicate that it is a suitable generic method to model yield surfaces for beam cross-sections. 
(a)

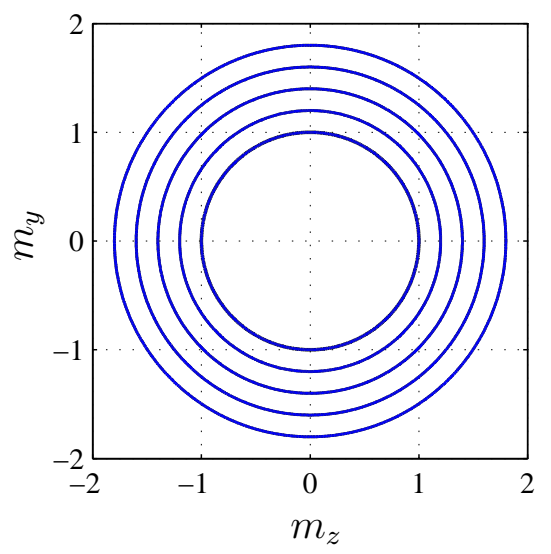

(b)

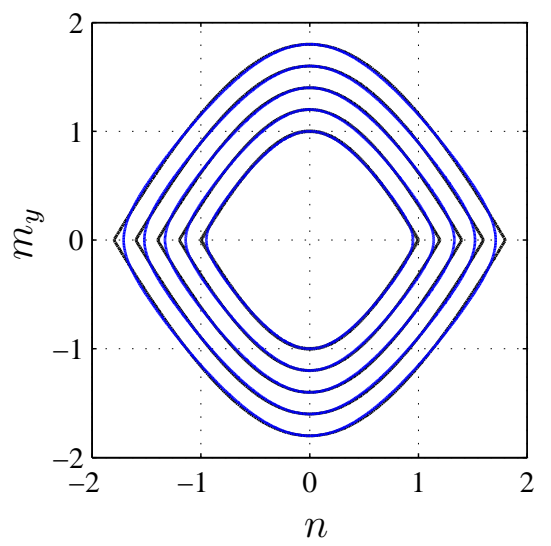

FIG. 5. Outer surfaces for accurate approximation, (-), and for rounded approximation, (-).

\section{BEAM MODEL WITH DEGRADING PLASTIC HINGES}

In principle, the plastic hinge model presented in Section 2 can be implemented for any type of element, where the generalized strains can be assumed to be the sum of elastic and plastic generalized strains. Many frame structures have loads leading to maximum load at joints or at specific cross-sections in the spans, and it is therefore of interest to use the theory for representing plastic deformation in the form of local yield hinges. In the following it will be demonstrated how the present cyclic plasticity model can be implemented in the form of a plastic hinge that is incorporated into the formulation of the element, leading to an explicit elasto-plastic tangent stiffness matrix including degradation effects. This beam element can then be used in a co-rotating element formulation for large displacements if needed. The formulation is an extension of that presented in (Krenk et al. 1999) to include the internal variable and damage features of the present plasticity model.

The beam element is formulated in terms of six equilibrium states and the conjugate states of deformation. It is assumed that the plastic deformation is concentrated at the nodes at the ends of the element in the form of yield hinges. With the choice of deformation measures for a beam shown in Fig. 6 the generalized elastic strains and the conjugate generalized stresses within the beam element between the plastic hinges are defined as

$$
\begin{aligned}
& \tilde{\mathbf{u}}_{\mathrm{e}}=\left[u, \varphi_{x}, \varphi_{z 1}, \varphi_{z 2}, \varphi_{y 1}, \varphi_{y 2}\right]^{T}, \\
& \tilde{\mathbf{q}}_{\mathrm{e}}=\left[N, T, M_{z 1}, M_{z 2}, M_{y 1}, M_{y 2}\right]^{T},
\end{aligned}
$$

where the tilde indicates reference to the six-component element equilibrium format. For the equilibrium beam element the generalized strains are the axial deformation and the rotations about the different axes, rather than the axial strain and the curvatures about 
(a)

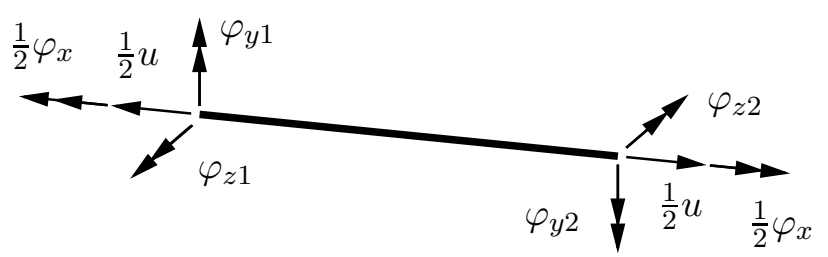

(b)

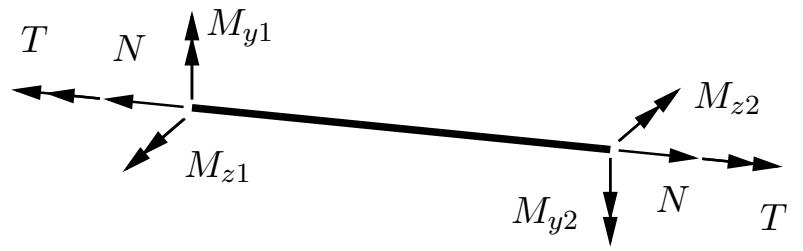

FIG. 6. Equilibrium format of beam element. (a) Element deformations, (b) section forces.

the different axes, in order to be conjugate to the section forces defined in (30). It is observed that in the equilibrium element the normal force and torsion moment are of equal magnitude but opposite at the two ends of the beam, and that the shear forces follow from the bending moments by equilibrium.

\section{Elasto-plastic tangent stiffness}

In the derivation of the elasto-plastic tangent stiffness it is necessary to have a relation between the elastic and the plastic deformations, which is particularly simple for small deformation theories which was applied in Section 2 as well. For a beam the assumption of small deformations, which can be combined with large displacements via e.g. the corotational formulation, implies that the total deformations, $\tilde{\mathbf{u}}_{\mathbf{t}}$, can be split additively into elastic deformation $\tilde{\mathbf{u}}_{\mathrm{e}}$ and plastic deformation $\tilde{\mathbf{u}}_{\mathrm{p}}$,

$$
\tilde{\mathbf{u}}_{\mathrm{t}}=\tilde{\mathbf{u}}_{\mathrm{e}}+\tilde{\mathbf{u}}_{\mathrm{p}}
$$

The internal energy of the beam element is completely analogous to the general format of the internal energy (2), with the only difference being the notation introduced to indicate the relation to a beam element,

$$
\Phi\left(\tilde{\mathbf{u}}_{\mathrm{e}}, \tilde{\mathbf{u}}_{\mathrm{i}}, \tilde{\mathbf{u}}_{\mathrm{d}}\right)=\frac{1}{2} \tilde{\mathbf{u}}_{\mathrm{e}}^{T} \mathbf{K}_{\mathrm{e}}\left(\tilde{\mathbf{u}}_{\mathrm{d}}\right) \tilde{\mathbf{u}}_{\mathrm{e}}+\frac{1}{2} \tilde{\mathbf{u}}_{\mathrm{i}}^{T} \mathbf{K}_{\mathrm{i}}\left(\tilde{\mathbf{u}}_{\mathrm{d}}\right) \tilde{\mathbf{u}}_{\mathrm{i}}+\Phi_{\mathrm{d}}\left(\tilde{\mathbf{u}}_{\mathrm{d}}\right)
$$

Whereas the original energy definition (2) was given per length the present energy applies to the full beam element. The energy is denoted $\Phi$ rather than $\varphi$, and the stiffness matrices are denoted $\mathbf{K}_{\mathrm{e}}$ and $\mathbf{K}_{\mathrm{i}}$ rather than $\mathbb{D}_{\mathrm{e}}$ and $\mathbb{D}_{\mathrm{i}}$, respectively. The energy conjugate section 
forces are found via differentiation as

$$
\begin{aligned}
& \tilde{\mathbf{q}}_{\mathrm{e}}=\partial_{\tilde{\mathbf{u}}_{\mathrm{e}}} \Phi=\mathbf{K}_{\mathrm{e}}\left(\tilde{\mathbf{u}}_{\mathrm{d}}\right) \tilde{\mathbf{u}}_{\mathrm{e}} \\
& \tilde{\mathbf{q}}_{\mathrm{i}}=\partial_{\tilde{\mathbf{u}}_{\mathrm{i}}} \Phi=\mathbf{K}_{\mathrm{i}}\left(\tilde{\mathbf{u}}_{\mathrm{d}}\right) \tilde{\mathbf{u}}_{\mathrm{i}} \\
& \tilde{\mathbf{q}}_{\mathrm{d}}=\partial_{\tilde{\mathbf{u}}_{\mathrm{d}}} \Phi
\end{aligned}
$$

The remaining steps of determining the elasto-plastic tangent stiffness matrix accounting for degradation follow the steps (8)-(17) using the generic yield surface described in Section 3. In order to retain a compact notation, the section forces $\tilde{\mathbf{q}}_{\mathrm{e}}$, the internal section forces $\tilde{\mathbf{q}}_{\mathbf{i}}$, and the section force-like damage parameters, $\tilde{\mathbf{q}}_{\mathbf{d}}$, are arranged in a common vector $\tilde{\mathbf{q}}$ that has the energy conjugate vector $\tilde{\mathbf{u}}$,

$$
\tilde{\mathbf{q}}^{T}=\left[\tilde{\mathbf{q}}_{\mathrm{e}}^{T}, \tilde{\mathbf{q}}_{\mathrm{i}}^{T}, \tilde{\mathbf{q}}_{\mathrm{d}}^{T}\right] \quad, \quad \tilde{\mathbf{u}}^{T}=\left[\tilde{\mathbf{u}}_{\mathrm{e}}^{T}, \tilde{\mathbf{u}}_{\mathrm{i}}^{T}, \tilde{\mathbf{u}}_{\mathrm{d}}^{T}\right]
$$

which are defined to ease the notation, especially when deriving the return algorithm.

The main difference between a beam element and the hinge model described in Section 2 is that plasticity may occur at each end of the beam individually, whereby the maximisation of the dissipation rate (11) will involve two constraints, namely the plastic flow potential at each end. The consistency condition for the yield surface at each end will provide the other constraints, making it possible to derive the elasto-plastic tangent stiffness. The plastic flow potentials and the yield functions are arranged in the vector format

$$
\mathbf{g}(\tilde{\mathbf{q}})=\left[G_{1}(\tilde{\mathbf{q}}), G_{2}(\tilde{\mathbf{q}})\right]^{T} \quad, \quad \mathbf{f}_{y}(\tilde{\mathbf{q}})=\left[F_{1}(\tilde{\mathbf{q}}), F_{2}(\tilde{\mathbf{q}})\right]^{T}
$$

With the use of the gradient of the plastic flow potential the increment in the total deformations, $d \tilde{\mathbf{u}}_{\mathrm{t}}$, internal deformations, $d \tilde{\mathbf{u}}_{\mathrm{i}}$ and deformation-like damage parameters, $d \tilde{\mathbf{u}}_{\mathrm{d}}$, can be expressed as

$$
\left[\begin{array}{c}
d \tilde{\mathbf{u}}_{\mathrm{t}} \\
\mathbf{0} \\
\mathbf{0}
\end{array}\right]=d \tilde{\mathbf{u}}+\left(\partial_{\tilde{\mathbf{q}}}^{T} \mathbf{g}\right)^{T} d \boldsymbol{\lambda} \quad, \quad d \boldsymbol{\lambda}=\left[\begin{array}{c}
d \lambda_{1} \\
d \lambda_{2}
\end{array}\right]
$$

Here $d \boldsymbol{\lambda}$ is the vector containing the two plastic multipliers, which are determined using the consistency condition at each end. The solution of the consistency condition is found in a compact form with use of the combined external, internal and damage stiffness matrices

$$
\begin{aligned}
& \mathbf{K}_{\mathrm{ed}}=\left[\begin{array}{lllll}
\mathbf{K}_{\mathrm{e}}\left(\tilde{\mathbf{u}}_{\mathrm{d}}\right), & \mathbf{0} & , & \partial_{\tilde{\mathbf{u}}_{\mathrm{d}}}^{T} \tilde{\mathbf{q}}_{\mathrm{e}}
\end{array}\right]^{T}, \\
& \mathbf{K}_{\text {eid }}=\left[\begin{array}{ccc}
\mathbf{K}_{\mathrm{e}}\left(\tilde{\mathbf{u}}_{\mathrm{d}}\right) & \mathbf{0} & \partial_{\tilde{\mathbf{u}}_{\mathrm{d}}}^{T} \tilde{\mathbf{q}}_{\mathrm{e}} \\
\mathbf{0} & \mathbf{K}_{\mathrm{i}}\left(\tilde{\mathbf{u}}_{\mathrm{d}}\right) & \partial_{\tilde{\mathbf{u}}_{\mathrm{d}}}^{T} \tilde{\mathbf{q}}_{\mathrm{i}} \\
\left(\partial_{\tilde{\mathbf{u}}_{\mathrm{d}}}^{T} \tilde{\mathbf{q}}_{\mathrm{e}}\right)^{T} & \left(\partial_{\tilde{\mathbf{u}}_{\mathrm{d}}}^{T} \tilde{\mathbf{q}}_{\mathrm{i}}\right)^{T} & \partial_{\tilde{\mathbf{u}}_{\mathrm{d}}}^{T} \tilde{\mathbf{q}}_{\mathrm{d}}
\end{array}\right],
\end{aligned}
$$


where $\mathbf{K}_{\text {ed }}$ is analogous to $\mathbb{D}_{\text {ed }}$ and $\mathbf{K}_{\text {eid }}$ is analogous to $\mathbb{D}_{\text {eid. }}$. The coupled evolution equations for the section forces $\tilde{\mathbf{q}}_{\mathrm{e}}$, the internal section forces $\tilde{\mathbf{q}}_{\mathrm{i}}$ and the section force-like damage parameters $\tilde{\mathbf{q}}_{\mathrm{d}}$ can compactly be described by

$$
d \tilde{\mathbf{q}}=\mathbf{K}_{\text {eid }} d \tilde{\mathbf{u}},
$$

which is essential in formulating a return algorithm as well as in solving the consistency conditions.

The solution of the consistency conditions gives the plastic multipliers

$$
d \boldsymbol{\lambda}=\left[\left(\partial_{\tilde{\mathbf{q}}}^{T} \mathbf{f}_{y}\right) \mathbf{K}_{\mathrm{eid}}\left(\partial_{\tilde{\mathbf{q}}}^{T} \mathbf{g}\right)^{T}\right]^{-1}\left(\partial_{\tilde{\mathbf{q}}}^{T} \mathbf{f}_{y}\right) \mathbf{K}_{\mathrm{ed}} d \tilde{\mathbf{u}}_{\mathrm{t}}
$$

where it is noted that $d \lambda_{j} \geq 0$ and the two equations reduce to one if the generalized stress state is only located on one of the two yield surfaces. The matrix product to be inverted is a $2 \times 2$ matrix in the case of yield at both nodes and reduces to a scalar in the case of yield at only one node. The increment in the section forces $d \tilde{\mathbf{q}}_{\mathrm{e}}$, the internal section forces $d \tilde{\mathbf{q}}_{\mathrm{i}}$, and the section force-like damage parameters, $d \tilde{\mathbf{q}}_{\mathrm{d}}$ are expressed as

$$
\begin{aligned}
d \tilde{\mathbf{q}} & =\left[d \tilde{\mathbf{q}}_{\mathrm{e}}^{T}, d \tilde{\mathbf{q}}_{\mathrm{i}}^{T}, d \tilde{\mathbf{q}}_{\mathrm{d}}^{T}\right]^{T} \\
& =\left[\mathbf{K}_{\mathrm{ed}}-\mathbf{K}_{\text {eid }}\left(\partial_{\tilde{\mathbf{q}}}^{T} \mathbf{g}\right)^{T}\left[\left(\partial_{\tilde{\mathbf{q}}}^{T} \mathbf{f}_{y}\right) \mathbf{K}_{\text {eid }}\left(\partial_{\tilde{\mathbf{q}}}^{T} \mathbf{g}\right)^{T}\right]^{-1}\left(\partial_{\tilde{\mathbf{q}}}^{T} \mathbf{f}_{y}\right) \mathbf{K}_{\mathrm{ed}}\right] d \tilde{\mathbf{u}}_{\mathrm{t}} .
\end{aligned}
$$

The tangent stiffness matrix relating only to the increment in the section forces, which is needed in the equilibrium iterations in a finite element code, is identified as

$$
\mathbf{K}_{\mathrm{ep}}=\mathbf{K}_{\mathrm{e}}-\mathbf{K}_{\mathrm{ed}}^{T}\left(\partial_{\tilde{\mathbf{q}}}^{T} \mathbf{g}\right)^{T}\left[\left(\partial_{\tilde{\mathbf{q}}}^{T} \mathbf{f}_{y}\right) \mathbf{K}_{\mathrm{eid}}\left(\partial_{\tilde{\mathbf{q}}}^{T} \mathbf{g}\right)^{T}\right]^{-1}\left(\partial_{\tilde{\mathbf{q}}}^{T} \mathbf{f}_{y}\right) \mathbf{K}_{\mathrm{ed}} .
$$

In the case without degradation of the elastic stiffness, the matrix $\mathbf{K}_{\mathrm{ed}}$ reduces to $\left[\mathbf{K}_{\mathrm{e}}, \mathbf{0}\right]^{T}$ and the elasto-plastic tangent stiffness is only affected by degradation in the hardening term, i.e. the matrix $\left[\left(\partial_{\tilde{\mathbf{q}}}^{T} \mathbf{f}_{y}\right) \mathbf{K}_{\text {eid }}\left(\partial_{\tilde{\mathbf{q}}}^{T} \mathbf{g}\right)^{T}\right]^{-1}$.

\section{Full-format beam element}

The $6 \times 6$ equilibrium format of the beam is not sufficient for an implementation in a finite element program as all rigid body displacements are absent. In order to be able to implement it in a finite element code the equilibrium beam element needs to be embedded in a general $12 \times 123 \mathrm{D}$ beam element as shown in Fig. 7 with the classical sign convention of the element displacements and the energy conjugate element forces.

Just as the deformations and section forces of the equilibrium element shown in Fig. 6 are arranged in the vectors $\tilde{\mathbf{u}}_{\mathrm{t}}$ and $\tilde{\mathbf{q}}_{\mathrm{e}}$, the element displacements and forces of the full format beam element shown in Fig. 7 are sorted in the vectors

$$
\begin{aligned}
& \mathbf{u}^{T}=\left[\mathbf{u}_{1}^{T}, \mathbf{u}_{2}^{T}\right] \quad, \quad \mathbf{u}_{j}=\left[u_{x}, u_{y}, u_{z}, \theta_{y z}, \theta_{x z}, \theta_{x y}\right]_{j}^{T}, \\
& \mathbf{q}^{T}=\left[\mathbf{q}_{1}^{T}, \mathbf{q}_{2}^{T}\right] \quad, \quad \mathbf{q}_{j}=\left[Q_{x}, Q_{y}, Q_{z}, M_{y z}, M_{x z}, M_{x y}\right]_{j}^{T} .
\end{aligned}
$$


(a)

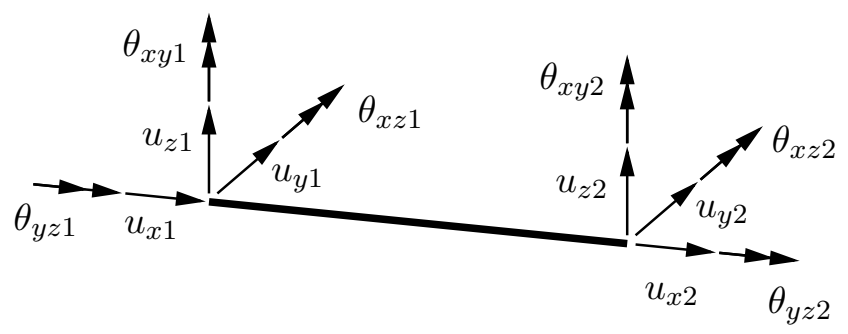

(b)

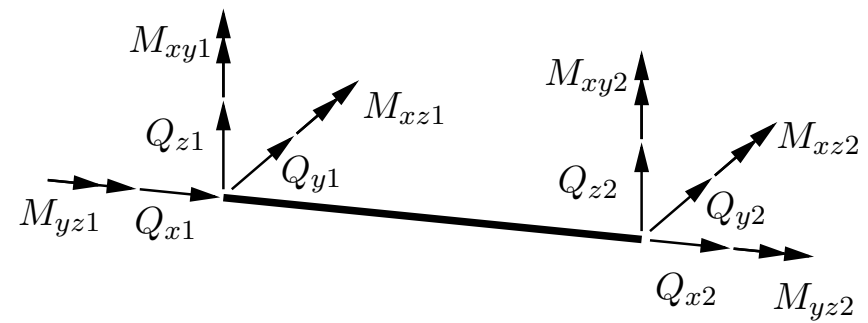

FIG. 7. Full format of beam element. (a) Element displacements, (b) element forces.

In order for the two elements to be equivalent the corresponding generalized strains and stresses of the two formulations must satisfy the incremental virtual work relation

$$
\mathbf{q}^{T} d \mathbf{u}=\tilde{\mathbf{q}}_{\mathrm{e}}^{T} d \tilde{\mathbf{u}}_{\mathrm{t}}
$$

providing a link between the two formulations. Via the equilibrium conditions of the beam the element forces $\mathbf{q}$ can be expressed in terms of the equilibrium section forces $\tilde{\mathbf{q}}_{\mathrm{e}}$ via a transformation matrix $\mathbf{T}$,

$$
\mathbf{q}=\left[\begin{array}{c}
\mathbf{q}_{1} \\
\mathbf{q}_{2}
\end{array}\right]=\left[\begin{array}{c}
\mathbf{T}_{1} \\
\mathbf{T}_{2}
\end{array}\right] \tilde{\mathbf{q}}_{\mathrm{e}}=\mathbf{T} \tilde{\mathbf{q}}_{\mathrm{e}}
$$

With this relation it is realised that the total deformations $\tilde{\mathbf{u}}_{\mathrm{t}}$ of the equilibrium format can be expressed via the nodal displacements $\mathbf{u}$ and the transformation matrix $\mathbf{T}$, using the work increment (47),

$$
d \tilde{\mathbf{u}}_{\mathrm{t}}=\mathbf{T}^{T} d \mathbf{u}
$$

With the use of (48) and (49) it is possible to derive the elasto-plastic tangent stiffness matrix for a beam element with 12 degrees of freedom as shown in Fig. 7. An increment in the element forces, $d \mathbf{q}$, can be expressed as

$$
d \mathbf{q}=\mathbf{T} d \tilde{\mathbf{q}}_{\mathrm{e}}+d \mathbf{T} \tilde{\mathbf{q}}_{\mathrm{e}}=\mathbf{T} \mathbf{K}_{\mathrm{ep}} \mathbf{T}^{T} d \mathbf{u}+d \mathbf{T} \tilde{\mathbf{q}}_{\mathrm{e}}
$$

The last term $d \mathbf{T} \tilde{\mathbf{q}}_{\mathrm{e}}$ relates to the geometric effects of rotation and length change of the element. While the second effect is small it contributes to the symmetry of the element stiffness matrix. The second term may be rearranged into the form of an additional 
stiffness matrix $\mathbf{K}_{r}$, see e.g. (Krenk et al. 1999; Krenk 2009),

$$
d \mathbf{q}=\left(\mathbf{T} \mathbf{K}_{\mathrm{ep}} \mathbf{T}^{T}+\mathbf{K}_{r}\right) d \mathbf{u},
$$

which has a form suitable for a finite element implementation, as it relates increments in the element forces directly to increments in the element displacements.

\section{Return algorithm}

In a finite element setting the use of the elastic or the elasto-plastic tangent stiffness matrix (44) will often lead to a violation of the yield criterion in the predicted state for finite load/deformation increments. In order to enable the use of larger increments, a return algorithm is developed which returns the predicted section force state to the yield surface taking into account kinematic hardening as well as the degradation mechanisms. The return algorithm is developed with the use of a Newton-Raphson solution scheme for the deformation evolution equation (38), using finite increments giving the residual

$$
\mathbf{r}_{\tilde{\mathbf{u}}}=\left[\begin{array}{c}
\Delta \tilde{\mathbf{u}}_{\mathrm{t}} \\
\mathbf{0} \\
\mathbf{0}
\end{array}\right]-\Delta \tilde{\mathbf{u}}-\left(\partial_{\tilde{\mathbf{q}}}^{T} \mathbf{g}\right)^{T} \Delta \boldsymbol{\lambda} \quad, \quad \Delta \boldsymbol{\lambda}=\left[\begin{array}{c}
\Delta \lambda_{1} \\
\Delta \lambda_{2}
\end{array}\right]
$$

The variation of the residual is needed in the Newton-Raphson solution procedure and because the yield function and the flow potential is defined via $\tilde{\mathbf{q}}$ it is desirable to formulate the return algorithm in terms of the subincrement of the section forces, $\delta \tilde{\mathbf{q}}$, rather than the subincrement of the deformations, $\delta \tilde{\mathbf{u}}$. However, the variation of the residual (52) is initially expressed via the subincrements $\delta \tilde{\mathbf{u}}, \delta \tilde{\mathbf{q}}$ and $\delta \boldsymbol{\lambda}$

$$
\delta \mathbf{r}_{\tilde{\mathbf{u}}}=-\delta \tilde{\mathbf{u}}-\partial_{\tilde{\mathbf{q}}}^{T}\left(\left(\partial_{\tilde{\mathbf{q}}}^{T} \mathbf{g}\right)^{T} \Delta \boldsymbol{\lambda}\right) \delta \tilde{\mathbf{q}}-\left(\partial_{\tilde{\mathbf{q}}}^{T} \mathbf{g}\right)^{T} \delta \boldsymbol{\lambda}
$$

because $\delta \tilde{\mathbf{u}}$ is rather conveniently expressed by $\delta \tilde{\mathbf{q}}$ via the variation of the evolution equation of $\tilde{\mathbf{q}},(41)$, yielding

$$
\delta \tilde{\mathbf{q}}=\left[\mathbf{K}_{\mathrm{eid}}+\partial_{\tilde{\mathbf{u}}}^{T}\left(\mathbf{K}_{\mathrm{eid}} \Delta \tilde{\mathbf{u}}\right)\right] \delta \tilde{\mathbf{u}} .
$$

In the Newton-Raphson scheme the increments $\Delta \tilde{\mathbf{u}}$ and $\Delta \boldsymbol{\lambda}$ are considered constants in each iteration and hence they are only placed inside the derivatives in (53) and (54) to give the correct dimension of vectors. Combining (52)-(54) with the consistency condition that the yield functions must be equal to zero, the following equation system defining the return algorithm is found,

$$
\left[\begin{array}{cc}
\left(\mathbf{K}_{\text {eid }}^{A}\right)^{-1} & \left(\partial_{\tilde{\mathbf{q}}}^{T} \mathbf{g}\right)^{T} \\
\partial_{\tilde{\mathbf{q}}}^{T} \mathbf{f}_{y} & \mathbf{0}
\end{array}\right]\left[\begin{array}{c}
\delta \tilde{\mathbf{q}} \\
\delta \boldsymbol{\lambda}
\end{array}\right]=\left[\begin{array}{c}
\mathbf{r}_{\tilde{\mathbf{u}}} \\
-\mathbf{f}_{y}
\end{array}\right],
$$

where the algorithmic stiffness matrix is defined via its inverse

$$
\left(\mathbf{K}_{\text {eid }}^{A}\right)^{-1}=\left[\mathbf{K}_{\text {eid }}+\partial_{\tilde{\mathbf{u}}}^{T}\left(\mathbf{K}_{\text {eid }} \Delta \tilde{\mathbf{u}}\right)\right]^{-1}+\partial_{\tilde{\mathbf{q}}}^{T}\left(\left(\partial_{\tilde{\mathbf{q}}}^{T} \mathbf{g}\right)^{T} \Delta \boldsymbol{\lambda}\right)
$$


The two derivatives needed in the algorithmic stiffness matrix are obtained as

$$
\partial_{\tilde{\mathbf{q}}}^{T}\left(\left(\partial_{\tilde{\mathbf{q}}}^{T} \mathbf{g}\right)^{T} \Delta \boldsymbol{\lambda}\right)=\frac{\partial^{2} G_{1}}{\partial \tilde{\mathbf{q}} \partial \tilde{\mathbf{q}}^{T}} \Delta \lambda_{1}+\frac{\partial^{2} G_{2}}{\partial \tilde{\mathbf{q}} \partial \tilde{\mathbf{q}}^{T}} \Delta \lambda_{2},
$$

and

$$
\partial_{\tilde{\mathbf{u}}}^{T}\left(\mathbf{K}_{\mathrm{eid}} \Delta \tilde{\mathbf{u}}\right)=\left[\begin{array}{ccc}
\frac{\partial^{2}\left(\tilde{\mathbf{q}}_{\mathrm{d}}^{T} \Delta \tilde{\mathbf{u}}_{\mathrm{d}}\right)}{\partial \tilde{\mathbf{u}}_{\mathrm{e}} \partial \tilde{\mathbf{u}}_{\mathrm{e}}^{T}} & \mathbf{0} & \frac{\partial^{2}\left(\tilde{\mathbf{q}}_{\mathrm{e}}^{T} \Delta \tilde{\mathbf{u}}_{\mathrm{e}}+\tilde{\mathbf{q}}_{\mathrm{d}}^{T} \Delta \tilde{\mathbf{u}}_{\mathrm{d}}\right)}{\partial \tilde{\mathbf{u}}_{\mathrm{d}} \partial \tilde{\mathbf{u}}_{\mathrm{e}}^{T}} \\
\frac{\partial^{2}\left(\tilde{\mathbf{q}}_{\mathrm{d}}^{T} \Delta \tilde{\mathbf{u}}_{\mathrm{d}}\right)}{\partial \tilde{\mathbf{u}}_{\mathrm{i}} \partial \tilde{\mathbf{u}}_{\mathrm{i}}^{T}} & \frac{\partial^{2}\left(\tilde{\mathbf{q}}_{\mathrm{i}}^{T} \Delta \tilde{\mathbf{u}}_{\mathrm{i}}+\tilde{\mathbf{q}}_{\mathrm{d}}^{T} \Delta \tilde{\mathbf{u}}_{\mathrm{d}}\right)}{\partial \tilde{\mathbf{u}}_{\mathrm{d}} \partial \tilde{\mathbf{u}}_{\mathrm{i}}^{T}} \\
\text { Sym. } & & \frac{\partial^{2}\left(\tilde{\mathbf{q}}_{\mathrm{e}}^{T} \Delta \tilde{\mathbf{u}}_{\mathrm{e}}+\tilde{\mathbf{q}}_{\mathrm{i}}^{T} \Delta \tilde{\mathbf{u}}_{\mathrm{i}}+\tilde{\mathbf{q}}_{\mathrm{d}}^{T} \Delta \tilde{\mathbf{u}}_{\mathrm{d}}\right)}{\partial \tilde{\mathbf{u}}_{\mathrm{d}} \partial \tilde{\mathbf{u}}_{\mathrm{d}}^{T}}
\end{array}\right] .
$$

$\partial_{\tilde{\mathbf{u}}}^{T}\left(\mathbf{K}_{\text {eid }} \Delta \tilde{\mathbf{u}}\right)$ is composed of three diagonal blocks, which themselves are symmetric matrices, as well as two off-diagonal blocks which are not symmetric. Because of the differentiation procedure the full matrix $\partial_{\tilde{\mathbf{u}}}^{T}\left(\mathbf{K}_{\text {eid }} \Delta \tilde{\mathbf{u}}\right)$ is symmetric though, as the off-diagonal blocks are transposed on the other side of the diagonal blocks.

Because both $\mathbf{K}_{\text {eid }}$ and $\partial_{\tilde{\mathbf{u}}}^{T}\left(\mathbf{K}_{\text {eid }} \Delta \tilde{\mathbf{u}}\right)$ have a structure with three symmetric diagonal blocks and two off-diagonal non-symmetric blocks it is possible to invert the first term, $\left[\mathbf{K}_{\text {eid }}+\partial_{\tilde{\mathbf{u}}}^{T}\left(\mathbf{K}_{\text {eid }} \Delta \tilde{\mathbf{u}}\right)\right]^{-1}$, of the inverse algorithmic stiffness matrix in a simple way as the sum $\mathbf{K}_{\text {eid }}+\partial_{\tilde{\mathbf{u}}}^{T}\left(\mathbf{K}_{\text {eid }} \Delta \tilde{\mathbf{u}}\right)$ has a block structure similar to $\mathbf{K}_{\text {eid }}$ and $\partial_{\tilde{\mathbf{u}}}^{T}\left(\mathbf{K}_{\text {eid }} \Delta \tilde{\mathbf{u}}\right)$. The inverse $\left[\mathbf{K}_{\text {eid }}+\partial_{\tilde{\mathbf{u}}}^{T}\left(\mathbf{K}_{\text {eid }} \Delta \tilde{\mathbf{u}}\right)\right]^{-1}$ can be determined using only the inverse of the two first diagonal blocks of the sum and the inverse of the difference between the third diagonal block of the sum and the matrix products of the off-diagonal blocks of the sum and the inverse of the corresponding diagonal block of the sum. The remaining part of inverting the first term of the inverse algorithmic stiffness matrix will then be simple matrix multiplication. The algorithmic stiffness matrix can therefore be determined by numerical inversion of one matrix which size depends on the amount of internal variables, $\tilde{\mathbf{u}}_{i}$, and damage variables, $\tilde{\mathbf{u}}_{\mathrm{d}}$, used in the element, along with inversion of three smaller matrices and a series of matrix multiplications. The two first block diagonal terms in the sum $\mathbf{K}_{\text {eid }}+\partial_{\tilde{\mathbf{u}}}^{T}\left(\mathbf{K}_{\text {eid }} \Delta \tilde{\mathbf{u}}\right)$ are most likely possible to invert analytically as they involve the elastic and internal stiffness matrices, which themselves tend to be block diagonal. It is furthermore noted that the algorithmic stiffness matrix is symmetric.

Using the algorithmic stiffness matrix (56) makes it possible to solve the equation system (55) in a relatively compact form without having to solve the full system numerically. Initially the force sub-increment $\delta \tilde{\mathbf{q}}$ is solved for in the first row of equations in (55) and subsequently substituted into the second row, making it possible to solve for $\delta \boldsymbol{\lambda}$, giving 
Calculate elastic increment in forces, $\Delta \tilde{\mathbf{q}}^{0}=\mathbf{K}_{\mathrm{ed}} \Delta \tilde{\mathbf{u}}_{\mathrm{t}}$.

1. Calculate value of yield functions, $\mathbf{f}_{y}^{k}$, using $\tilde{\mathbf{q}}=\tilde{\mathbf{q}}^{0}+\Delta \tilde{\mathbf{q}}^{k}$.

If $F_{1}^{0} \leq 0$ and $F_{2}^{0} \leq 0$, accept $\tilde{\mathbf{q}}=\tilde{\mathbf{q}}^{0}+\Delta \tilde{\mathbf{q}}^{0}$

and exit algorithm.

2. Calculate $\Delta \boldsymbol{\lambda}^{0}$ using (42) and $\Delta \tilde{\mathbf{u}}_{\mathrm{t}}$

or update $\Delta \boldsymbol{\lambda}^{k}=\Delta \boldsymbol{\lambda}^{k-1}+\delta \boldsymbol{\lambda}^{k-1}$.

3. Determine $\partial_{\tilde{\mathbf{q}}}^{T} \mathbf{g}, \partial_{\tilde{\mathbf{q}}}^{T} \mathbf{f}_{y}$ and $\mathbf{r}_{\tilde{\mathbf{u}}}^{k}$ using $\tilde{\mathbf{q}}=\tilde{\mathbf{q}}^{0}+\Delta \tilde{\mathbf{q}}^{k}$.

If $\left\|\mathbf{r}_{\tilde{\mathbf{u}}}^{k}\right\|<\epsilon_{t o l}$, accept $\tilde{\mathbf{q}}=\tilde{\mathbf{q}}^{0}+\Delta \tilde{\mathbf{q}}^{k}$ and exit algorithm.

4. Calculate $\mathbf{K}_{\text {eid }}^{A}$ by (56) and determine $\delta \boldsymbol{\lambda}^{k}$ by (59).

5. Determine subincrements $\delta \tilde{\mathbf{q}}^{k}$ and $\delta \tilde{\mathbf{u}}^{k}$ by (60) and (54).

6. Update $\Delta \tilde{\mathbf{q}}^{k+1}=\Delta \tilde{\mathbf{q}}^{k}+\delta \tilde{\mathbf{q}}^{k}$ and $\Delta \tilde{\mathbf{u}}^{k+1}=\Delta \tilde{\mathbf{u}}^{k}+\delta \tilde{\mathbf{u}}^{k}$

and go to 1 using $k=k+1$.

the relations

$$
\begin{aligned}
& \delta \boldsymbol{\lambda}=\left[\left(\partial_{\tilde{\mathbf{q}}}^{T} \mathbf{f}_{y}\right) \mathbf{K}_{\text {eid }}^{A}\left(\partial_{\tilde{\mathbf{q}}}^{T} \mathbf{g}\right)^{T}\right]^{-1}\left(\left(\partial_{\tilde{\mathbf{q}}}^{T} \mathbf{f}_{y}\right) \mathbf{K}_{\text {eid }}^{A} \mathbf{r}_{\tilde{\mathbf{u}}}+\mathbf{f}_{y}\right), \\
& \delta \tilde{\mathbf{q}}=\mathbf{K}_{\text {eid }}^{A}\left(\mathbf{r}_{\tilde{\mathbf{u}}}-\left(\partial_{\tilde{\mathbf{q}}}^{T} \mathbf{g}\right)^{T} \delta \boldsymbol{\lambda}\right) .
\end{aligned}
$$

The return algorithm described by (52) and (56)-(60) is shown as pseudo-code in Table 1.

The return algorithm is based on a fixed deformation increment for each element, $\Delta \tilde{\mathbf{u}}_{t}$, which is obtained via a displacement increment found in a global finite element analysis which is subsequently transformed into a deformation increment for each element via (49). Additionally, the section forces prior to the deformation increment $\tilde{\mathbf{q}}^{0}$ is known. Initially an elastic prediction step of $\Delta \tilde{\mathbf{q}}$ is made and if the new stress state is located on or inside the yield surface, the elastic prediction step is accepted as an elastic change has taken place, because of the convexity of the yield surface. Subsequently the increment in the plastic multipliers $\Delta \boldsymbol{\lambda}^{0}$ is calculated, based on the finite increment in deformations $\Delta \tilde{\mathbf{u}}_{\mathrm{t}}$, followed by determination of the gradients of the plastic flow potential and the yield surface, $\partial_{\tilde{\mathbf{q}}}^{T} \mathbf{g}$ and $\partial_{\tilde{\mathbf{q}}}^{T} \mathbf{f}_{y}$, respectively, along with the current value of the residual $\mathbf{r}_{\tilde{\mathbf{u}}}^{k}$ based on the predicted final value of $\tilde{\mathbf{q}}$. If the residual is sufficiently small, the iteration procedure is ended, while otherwise the algorithmic stiffness matrix $\mathbf{K}_{\text {eid }}^{A}$ is recalculated via (56) and the sub-increment in the plastic multipliers is calculated, $\delta \boldsymbol{\lambda}^{k}$. There are limitations on 
the values $\delta \boldsymbol{\lambda}^{k}$, as $\Delta \lambda_{j} \geq 0$ for $j=1,2$. The subincrements $\delta \tilde{\mathbf{q}}^{k}$ and $\delta \tilde{\mathbf{u}}^{k}$ are determined via (60) and (54) respectively, whereby the increments $\Delta \tilde{\mathbf{q}}^{k}$ and $\Delta \tilde{\mathbf{u}}^{k}$ can be updated and the iteration procedure repeated until convergence.

\section{A simple damage formulation}

A very simple damage formulation is implemented in the beam element to illustrate the concept of degradation. The damage functions used for the different parameters are chosen because of the ability to model increase as well as decrease of a parameter with a prescribed asymptotic saturation level, and because of their simple format. In total 9 different damage variables are introduced, one relating to the elastic stiffness and two relating to the inelastic stiffness, the yield capacity, the $\beta_{j}$ values and the $\alpha_{j}$ values, respectively. The deformationlike damage parameters are arranged in the vector

$$
\tilde{\mathbf{u}}_{\mathrm{d}}=\left[\tilde{u}_{\mathrm{d}}^{\mathrm{e}}, \tilde{u}_{\mathrm{d}}^{\mathrm{i}, 1}, \tilde{u}_{\mathrm{d}}^{\mathrm{i}, 2}, \tilde{u}_{\mathrm{d}}^{\mathrm{y}, 1}, \tilde{u}_{\mathrm{d}}^{\mathrm{y}, 2}, \tilde{u}_{\mathrm{d}}^{\beta, 1}, \tilde{u}_{\mathrm{d}}^{\beta, 2}, \tilde{u}_{\mathrm{d}}^{\alpha, 1}, \tilde{u}_{\mathrm{d}}^{\alpha, 2}\right]^{T}
$$

where the superscript indicates what parameter type and node number the damage variable relates to. The elastic stiffness is degraded via the function

$$
\mathbf{K}_{\mathrm{e}}\left(\tilde{\mathbf{u}}_{\mathrm{d}}\right)=\frac{1+\eta_{\mathrm{e}} \tilde{u}_{\mathrm{d}}^{\mathrm{e}} / \tilde{u}_{\mathrm{d}, 0}^{\mathrm{e}}}{1+\tilde{u}_{\mathrm{d}}^{\mathrm{e}} / \tilde{u}_{\mathrm{d}, 0}^{\mathrm{e}}} \mathbf{K}_{\mathrm{e}}^{0}
$$

where $\mathbf{K}_{\mathrm{e}}^{0}$ is the elastic stiffness matrix without damage. $\eta_{e}$ is the saturation level and $\tilde{u}_{\mathrm{d}, 0}^{\mathrm{e}}$ is a factor accounting for how fast the saturation level is reached. Similarly, the stiffness matrix relating to the deformation-like internal parameters is degraded via the function

$$
\mathbf{K}_{\mathrm{i}}\left(\tilde{\mathbf{u}}_{\mathrm{d}}\right)=\left[\begin{array}{cc}
\mathbf{K}_{\mathrm{i}}^{1}\left(\tilde{\mathbf{u}}_{\mathrm{d}}\right) & \mathbf{0} \\
\mathbf{0} & \mathbf{K}_{\mathrm{i}}^{2}\left(\tilde{\mathbf{u}}_{\mathrm{d}}\right)
\end{array}\right] \quad, \quad \mathbf{K}_{\mathrm{i}}^{j}\left(\tilde{\mathbf{u}}_{\mathrm{d}}\right)=\frac{1+\eta_{\mathrm{i}, j} \tilde{u}_{\mathrm{d}}^{\mathrm{i}, j} / \tilde{u}_{\mathrm{d}, 0}^{\mathrm{i}, j}}{1+\tilde{u}_{\mathrm{d}}^{\mathrm{i}, j} / \tilde{u}_{\mathrm{d}, 0}^{\mathrm{i}, j}} \mathbf{K}_{\mathrm{i}, j}^{0},
$$

where $\mathbf{K}_{\mathrm{i}}^{j}$ is the part of the internal stiffness matrix relating to node $j$ with the corresponding undamaged internal stiffness matrix $\mathbf{K}_{\mathbf{i}, j}^{0}$. The remaining material parameters are functions of the section force-like damage parameters $\tilde{\mathbf{q}}_{\mathrm{d}}$, and each of these are defined as

$$
\tilde{q}_{\mathrm{d}}^{k}=\frac{1+\eta_{k, j} \tilde{u}_{\mathrm{d}}^{k, j} / \tilde{u}_{\mathrm{d}, 0}^{k, j}}{1+\tilde{u}_{\mathrm{d}}^{k, j} / \tilde{u}_{\mathrm{d}, 0}^{k, j}},
$$

where the index $k$ may be $y, \beta$ or $\alpha$. The relation between the material parameters and the section force-like damage parameters are defined as

$$
\mathbb{B}_{y, j}\left(\tilde{\mathbf{q}}_{\mathrm{d}}\right)=\tilde{q}_{\mathrm{d}}^{y, j} \mathbb{B}_{y, j}^{0} \quad, \quad \boldsymbol{\beta}_{j}\left(\tilde{\mathbf{q}}_{\mathrm{d}}\right)=\tilde{q}_{\mathrm{d}}^{\beta, j} \boldsymbol{\beta}_{j}^{0} \quad, \quad \boldsymbol{\alpha}_{j}\left(\tilde{\mathbf{q}}_{\mathrm{d}}\right)=\tilde{q}_{\mathrm{d}}^{\alpha, j} \boldsymbol{\alpha}_{j}^{0},
$$

where $\mathbb{B}_{y, j}^{0}$ is the undamaged yield capacity matrix for node $j, \boldsymbol{\beta}_{j}^{0}$ is the array of undamaged $\beta$-values for node $j$, and $\boldsymbol{\alpha}_{j}^{0}$ is the array of undamaged $\alpha$-values for node $j$. The damage functions described above are used in the following examples. 


\section{EXAMPLES}

The element described in Section 4 combined with the plastic hinge presented in Section 2 and the generic yield surface described in Section 3 is implemented in a MATLAB toolbox, CycPlasframe. The toolbox has linear geometry and does not at the present stage include the stiffness contribution from normal forces, making it non-suitable for frame structures dominated by buckling failure but highly suitable for frame structures dominated by plastic deformation.

\section{Cantilevered I-beams}

Estimates of the model parameters can be found by use of experimental results and most easily with displacement controlled experiments of cantilevered beams. Displacement controlled bending of a cantilevered beam activates only one hinge, eliminating the coupling between hinges and subsequently simplifying the calibration procedure. Cantilevered Ibeams with various cross-sections subjected to cyclic displacement controlled bending have been tested in (D'Aniello et al. 2012), where a significant degradation of the parameters is observed, primarily because of local buckling. In the test a cantilever of length $L=1.875 \mathrm{~m}$ is subjected to 12 symmetric cycles with approximate tip displacement $2 \cdot 10^{-3} \mathrm{~L}$ followed by six symmetric cycles of approximate tip displacement $3.5 \cdot 10^{-3} \mathrm{~L}$, four symmetric cycles of approximate tip displacement $5 \cdot 10^{-3} \mathrm{~L}$ and subsequently by sets of two symmetric cycles where the tip displacement increased by approximately $10^{-2} \mathrm{~L}$ per set until failure. Test results for an IPE 300 cross-section and a HEB 240 cross-section are here used for calibration of the present model. The IPE 300 and HEB 240 have approximately the same slenderness of the flanges but the web of the IPE 300 is substantially more slender than the web of the HEB 240, suggesting that local buckling and thereby degradation will occur earlier in the bending of the IPE 300 than the HEB 240. The model parameters for the non-degraded system are presented in Table 2.

TABLE 2. Initial model parameters.

\begin{tabular}{lccccc}
\hline Cross-section & $\left(E I_{y}\right)_{\mathrm{e}}\left[\mathrm{MNm}^{2}\right]$ & $\frac{\left(E I_{y}\right)_{\mathrm{i}}}{\left(E I_{y}\right)_{\mathrm{e}}}[-]$ & $M_{y}^{y}[\mathrm{kNm}]$ & $\beta_{M_{y}}[-]$ & $\alpha_{M_{y}}[-]$ \\
\hline IPE 300 & 15.9 & 4.5 & 175.8 & 0.20 & 0.80 \\
HEB 240 & 21.4 & 2.0 & 295.1 & 0.03 & 0.80 \\
\hline
\end{tabular}

It is observed in Table 2 that aside from being more slender, the IPE 300 also has a smaller elastic stiffness and a smaller yield capacity than the HEB 240 profile. The model parameters relating to degradation of stiffness is shown in Table 3.

The higher slenderness of the IPE 300 profile compared to the HEB 240 profile shows in the degradation parameters in Table 3 as $u_{\mathrm{d}, 0}^{\mathrm{e}}$ and $u_{\mathrm{d}, 0}^{\mathrm{i}, 1}$ are significantly lower for the 
TABLE 3. Model parameters for stiffness degradation.

\begin{tabular}{lcccc}
\hline Cross-section & $u_{\mathrm{d}, 0}^{\mathrm{e}}[\mathrm{kJ}]$ & $\eta_{\mathrm{e}}[-]$ & $u_{\mathrm{d}, 0}^{\mathrm{i}, 1}[\mathrm{~kJ}]$ & $\eta_{\mathrm{i}, 1}[-]$ \\
\hline IPE 300 & 60 & 0.2 & 28 & 0.3 \\
HEB 240 & 400 & 0.2 & 70 & 0.4 \\
\hline
\end{tabular}

TABLE 4. Model parameters for capacity degradation.

\begin{tabular}{lcccccc}
\hline Cross-section & $u_{\mathrm{d}, 0}^{y, 1}[\mathrm{~kJ}]$ & $\eta_{y, 1}[-]$ & $u_{\mathrm{d}, 0}^{\beta, 1}[\mathrm{~kJ}]$ & $\eta_{\beta, 1}[-]$ & $u_{\mathrm{d}, 0}^{\alpha, 1}[\mathrm{~kJ}]$ & $\eta_{\alpha, 1}[-]$ \\
\hline IPE 300 & 70 & 0.3 & 0.30 & 7.8 & -70 & 1.10 \\
HEB 240 & 700 & 0.2 & 0.80 & 95 & -100 & 1.06 \\
\hline
\end{tabular}

IPE 300 profile, indicating that the degradation process develops in a faster rate. The increment in the deformation-like damage parameters $u_{\mathrm{d}}^{\mathrm{e}}$ and $u_{\mathrm{d}}^{\mathrm{i}, 1}$ are proportional to $d \lambda_{1}$ and thus have dimension of energy and relate to the dissipated energy. This suggests that the reference values $u_{\mathrm{d}, 0}^{\mathrm{e}}$ and $u_{\mathrm{d}, 0}^{\mathrm{i}, 1}$ may be scaled with the size of a characteristic hysteresis loop for cross-sections of similar type. The model parameters for degradation of the capacity parameters and $\alpha$ are shown in Table 4 . Similar to Table 3 it is observed that the reference energy levels, $u_{\mathrm{d}, 0}^{y, 1}, u_{\mathrm{d}, 0}^{\beta, 1}$ and $u_{\mathrm{d}, 0}^{\alpha, 1}$ are lower for the IPE 300 profile compared to the HEB 240 profile.

(a)

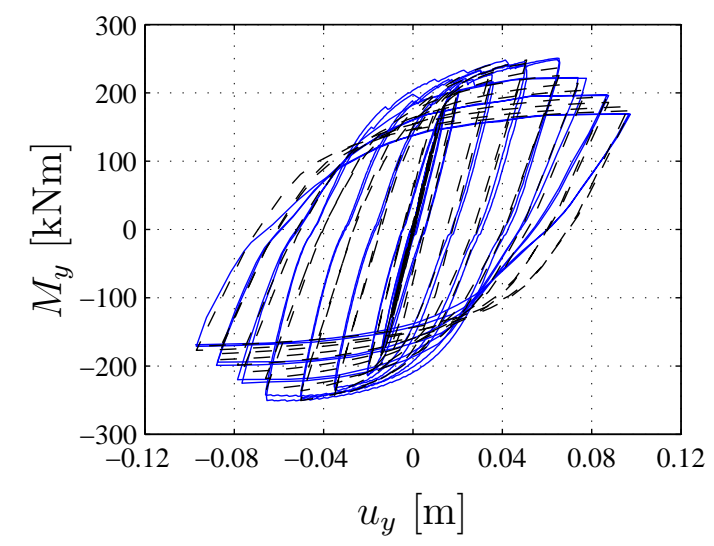

(b)

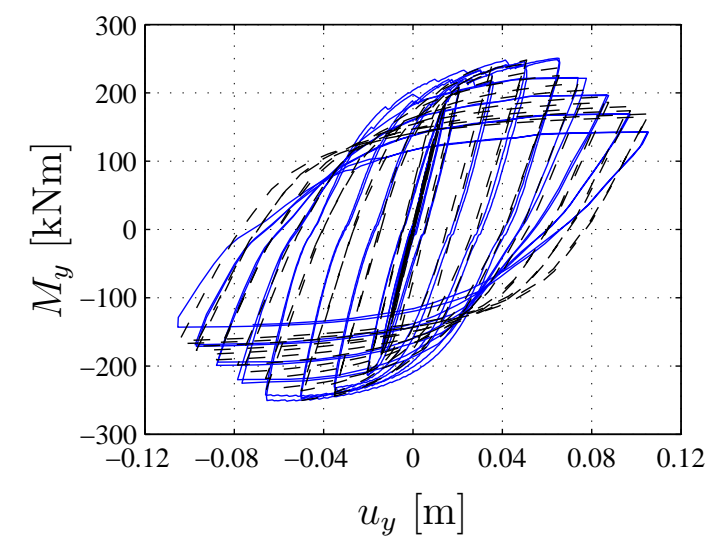

FIG. 8. Cyclic bending of cantilevered IPE 300 beam: experiment (D'Aniello et al. 2012): (-). Present model (- -). (a) First 38 cycles. (b) All 40 cycles.

Both the experimental and the modelled results of the tip displacement and reaction moment of the IPE 300 is plotted in Fig. 8. In Fig. 8 (a) the first 38 cycles are shown, to illustrate that the accuracy of the model is fairly high for most of the response despite the very simple damage functions used. In Fig. 8 (b) the remaining 2 cycles are also included, 
and it is observed that the last two cycles are not modelled very accurately compared to the previous cycles. In the experiment torsional buckling took place after the local plastic buckling (D'Aniello et al. 2012), which lead to failure of the beam, and it is expected that this mechanism is the cause of the non-smooth change in the response in the last two cycles compared to the initial $\sim 38$ cycles.

(a)

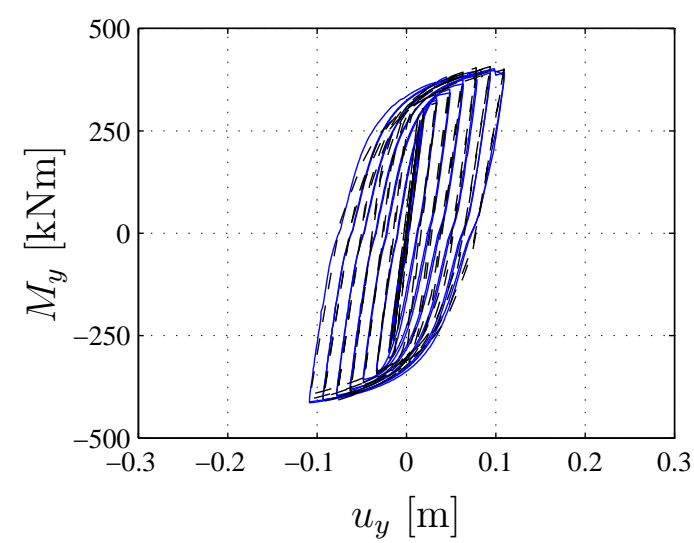

(c)

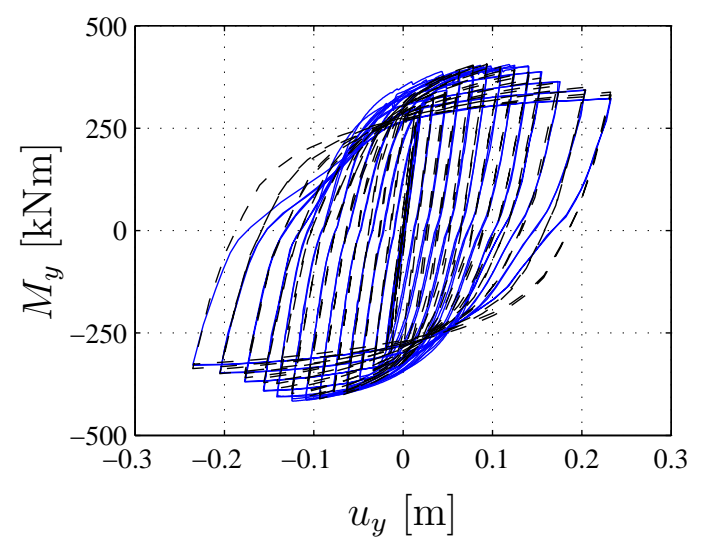

(b)

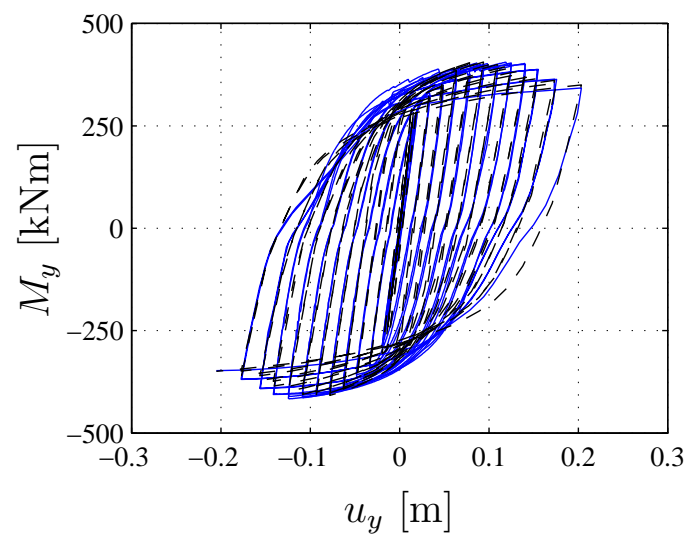

(d)

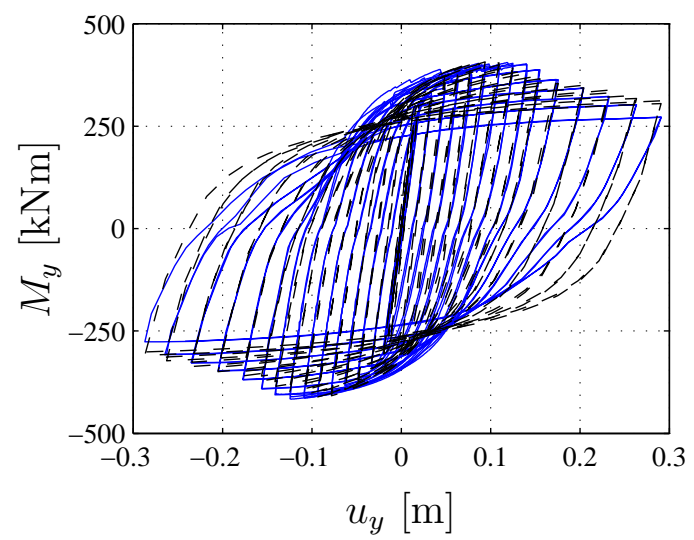

FIG. 9. Cyclic bending of cantilevered HEB 240 beam: experiment (D'Aniello et al. 2012): (-). Present model (- ). (a) First 38 cycles. (b) First 47 cycles. (c) First 50 cycles. (d) All 54 cycles.

The response of the HEB 240 profile is shown in Fig. 9. It is observed in Fig. 9 (a) that the first 38 cycles are modelled almost perfectly with a very little difference between the experimental and modelled result. In the first 38 cycles very little degradation takes place, suggesting that the model without degradation is sufficiently accurate. Comparing Fig. 9 (b) to (a) it is observed that after an additional nine cycles, during which a significant amount of degradation has occurred, the modelled response still represents the experimental response with good accuracy, despite the simple damage functions used. When observing Fig. 9 (c) and (d) it is found that once the degradation process progresses further the modelled response is not as accurate as for the first cycles. Torsional buck- 
ling also occurred in the final stages of the experimental testing of the HEB 240 profile (D'Aniello et al. 2012), which might explain the discrepancy between the experimental and modelled response as in the case of the IPE 300 profile.

\section{Tubular offshore frame}

The plastic hinge model has been implemented as an extension of the computer code RONJA (Rambøll Offshore Nonlinear Jacket Analysis) using a finite displacement corotational beam-column formulation, (Krenk et al. 1999). This implementation is here used to model cyclic loading on a plane offshore frame structure shown in Fig. 10 and tested by (Zayas et al. 1980). This structure is geometrically similar to that analyzed in (Krenk et al. 1999) for monotonic loading by a linear hardening plasticity model, but representation of the response to cyclic loading is a considerably more challenging task. The jacket structure is a one-to-six model, representative of offshore jacket structures located in the Mexican Gulf. The jacket has equal bay heights and width of $3.05 \mathrm{~m}$ and is simply supported at the bottom of the legs and loaded by an in-plane horizontal compression force $P$ at the top right corner. Each part of the bracing is modelled by two beam-column elements. Plastic hinges develop in the lower left brace of the upper bay, and in order to capture the column effect a suitable imperfection is introduced in this brace by a center node offset in the transverse direction of $56 \mathrm{~mm}$, corresponding to $2.6 \%$. The remaining members do not buckle and are initially straight. The legs of the jacket have an outer diameter of $324 \mathrm{~mm}$ and a thickness of $9.53 \mathrm{~mm}$, while all horizontal bracing and the upper bay bracing has outer diameter $102 \mathrm{~mm}$ and thickness $3.05 \mathrm{~mm}$. The lower bay bracing have outer diameter $114 \mathrm{~mm}$ and thickness $4.78 \mathrm{~mm}$, while the top half bracing has outer diameter $152 \mathrm{~mm}$ and thickness $4.60 \mathrm{~mm}$.

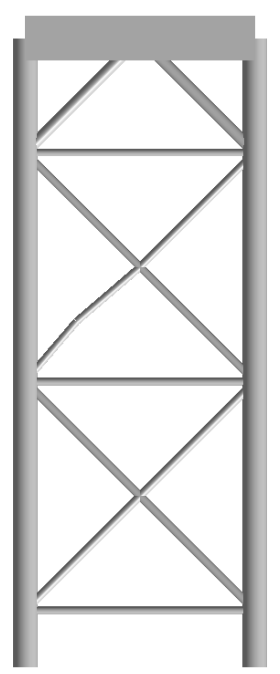

FIG. 10. Plane offshore frame structure geometry. 
The steel in the bracing has yield stress $180 \mathrm{MPa}$ and the legs have yield stress $300 \mathrm{MPa}$. All members are modelled with $\beta_{N}=\beta_{M_{y}}=\beta_{M_{z}}=0.700$ and $\alpha_{N}=\alpha_{M_{y}}=\alpha_{M_{z}}=0.90$. The relative internal stiffness of the bracing is $(E A)_{\mathrm{i}} /(E A)_{\mathrm{e}}=0.6$ and $(E I)_{\mathrm{i}} /(E I)_{\mathrm{e}}=2.0$. The legs have sufficient strength to avoid the formation of plastic hinges. As the $D / t$-ratio of all members are relatively low, local buckling is not expected and degradation effects are not included in the model. The horizontal load-displacement, $P-\Delta u$, curve of the loaded top right corner is shown in Fig. 11(a,b,c), with each sub-figure showing five cycles for clarity.

(a)

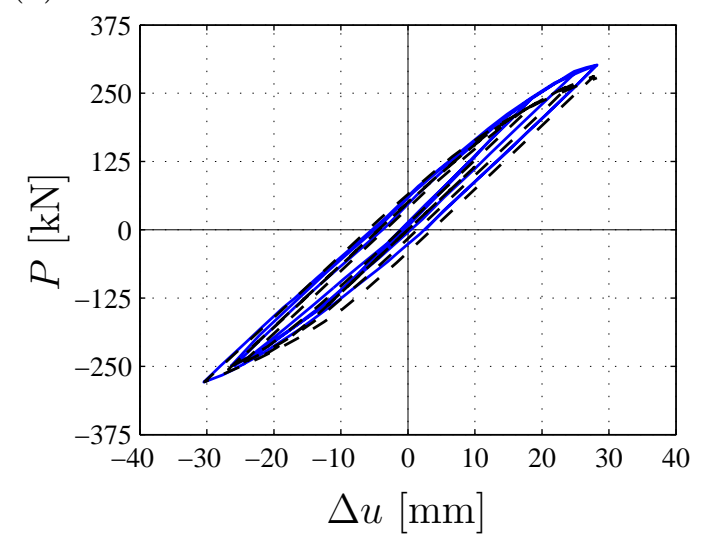

(b)

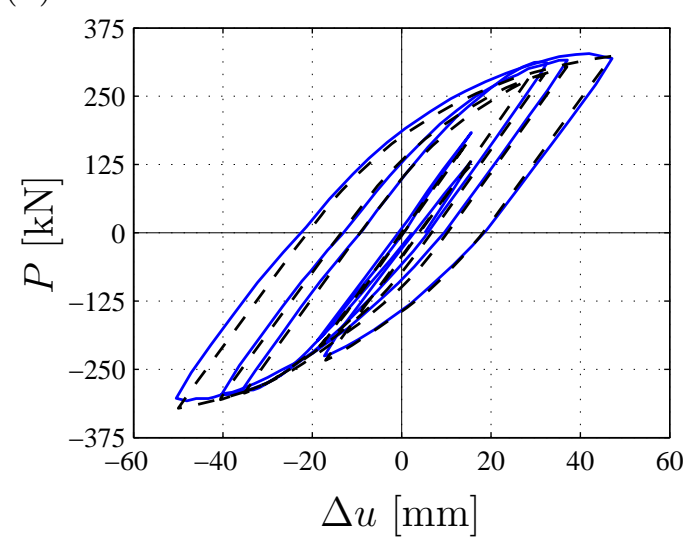

(c)

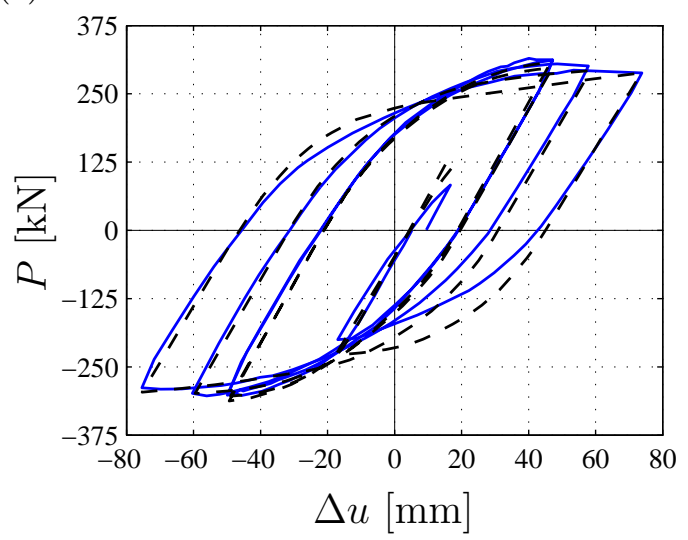

FIG. 11. Plane offshore frame: experiment (Zayas et al. 1980) (-). Present model (- -). (a) Cycle 1-5, (b) Cycle 6-10, (c) Cycle 11-15.

The figure shows good agreement between the experiment and the results of the model, and the characteristic load levels and shapes of the hysteresis curves of the model replicate the experimental results fairly well. It is noted that a degradation of the ultimate capacity of the structure is observed, caused by the permanent deformation of the geometry of the structure originating from elasto-plastic deformation and column-buckling.

\section{CONCLUSIONS}

A model of cyclic plastic hinges based on generalized stresses in the form of section forces 
has been presented. The model has the ability to degrade stiffness and strength to account for the effects of local buckling, fracture etc. in frame structures. The model is based on a yield surface and a plastic flow potential which have the same gradient with respect to the section forces. For beams typical analytical yield surfaces have corners which are numerically difficult to handle and a generic, smooth and convex yield surface representation is suggested. The present yield surface is formulated in terms of normalized generalized stresses and is homogeneous of degree one which in combination with the plastic flow potential ensures the desired plastic behaviour.

The plastic hinge model is described by five parameters for each generalized stress component; the elastic and elasto-plastic stiffness, the yield and ultimate capacity and a parameter controlling the shape of the hysteresis curve between yield and ultimate capacity. The plastic hinge model has been implemented in an elasto-plastic beam element formulated via an equilibrium format whereby the stiffness format can be inverted to the flexibility format which is additive. A return algorithm is formulated for the beam element via the flexibility format whereby the consistent algorithmic tangent stiffness matrix is derived. The algorithmic tangent stiffness matrix accounts for change in stiffness during yielding as well as change of stiffness and capacity parameters leading to an efficient return algorithm.

The element has been used to model cyclic bending of cantilevered steel beams utilizing very simple damage functions. In the final stages of the experiment degradation included local instability effects, the representation of which will need further refinement of the specific damage model. The computed results compare well with the experimental results. The model has also been used to model and calculate the response of a tubular offshore frame to cyclic loading. Also in this case the model captures the response well and clearly represents the experimentally observed increased displacements in continued cycling.

\section{ACKNOWLEDGEMENTS}

This paper is part of a project sponsored jointly by the Danish Agency for Science, Technology and Innovation, Maersk Oil A/S and the Technical University of Denmark. The authors gratefully acknowledge access to the digital data of (D'Aniello et al. 2012), provided by Prof. Raffaele Landolfo.

\section{REFERENCES}

Attalla, M. R., Deierlein, G. G., and McGuire, W. (1994). "Spread of plasticity: Quasiplastic-hinge approach." Journal of Structural Engineering, 120, 2451-2473.

Baber, T. T. and Wen, Y.-K. (1981). "Random vibration of hysteretic, degrading systems." Journal of the Engineering Mechanics Division, ASCE, 107, 1069-1087. 
Bleyer, J. and de Buhan, P. (2013a). "A greedy algorithm for yield surface approximation." Comptes Rendus Mecanique, 341, 605-615.

Bleyer, J. and de Buhan, P. (2013b). "Yield surface approximation for lower and upper bound yield design of 3D composite frame structures." Computers \& Structures, 129, $86-98$.

Chen, W.-F. and Atsuta, T. (2008). Theory of Beam-Columns. J. Ross Publishing, Fort Lauderdale, USA.

Cipollina, A., López-Inojosa, A., and Flórez-López, J. (1995). "A simplified damage mechanics approach to nonlinear analysis of frames." Computers \& Structures, 54, 11131126 .

Coombs, W. M., Petit, O. A., and Motlagh, Y. G. (2016). "NURBS plasticity: yield surface representation and implicit stress integration for isotropic inelasticity." Computer Methods in Applied Mechanics and Engineering, 304, 342-358.

D'Aniello, M., Landolfo, R., Piluso, V., and Rizzano, G. (2012). "Ultimate behavior of steel beams under non-uniform bending." Journal of Constructional Steel Research, 78, $144-158$.

Elchalakani, M. (2007). "Plastic mechanism analyses of circular tubular members under cyclic loading." Thin-Walled Structures, 45, 1044-1057.

Elchalakani, M., Zhao, X. L., and Grzbieta, R. (2003). "Tests of cold-formed circular tubular braces under cyclic axial loading." Journal of Structural Engineering, 129, 507514.

Folino, P., Etse, G., and Will, A. (2009). "Performance dependent failure criterion for normal- and high-strength concretes." Journal of Engineering Mechanics, ASCE, 135, 1393-1409.

Ibarra, L. F., Medina, R. A., and Krawinkler, H. (2005). "Hysteretic models that incorporate strength and stiffness deterioration." Earthquake Engineering and Structural Dynamics, 34, 1489-1511.

Inglessis, P., Gómez, G., Quintero, G., and Flórez-López, J. (1999). "Model of damage for steel frame members." Engineering Structures, 21, 954-964.

Iu, C. K. and Chan, S. L. (2004). "A simulation-based large deflection and inelastic analysis of steel frames under fire." Journal of Constructional Steel Research, 60, 1495-1524.

Jiang, X.-M., Chen, H., and Liew, J. Y. R. (2002). "Spread-of-plasticity analysis of threedimensional steel frames." Journal of Constructional Steel Research, 58, 193-212.

Kaewkulchai, G. and Williamson, E. B. (2004). "Beam element formulation and solution procedure for dynamic progressive collapse analysis." Computers \& Structures, 82, 639651.

Kamaris, G. S., Hatzigeorgiou, G. D., and Beskos, D. E. (2013). "A new damage index for plane steel frames exhibiting strength and stiffness degradation under seismic motion." 
Engineering Structures, 46, 727-736.

Kitipornchai, S., Zhu, K., Xiang, Y., and Al-Bermani, F. G. A. (1991). "Single-equation yield surfaces for monosymmetric and asymmetric sections." Engineering Structures, 13, 366-370.

Krenk, S. (2009). Non-Linear Modeling and Analysis of Solids and Structures. Cambridge University Press, Cambridge, UK.

Krenk, S. and Tidemann, L. (2016). "Cyclic plasticity model with degradation effects." Submitted for publication.

Krenk, S., Vissing, S., and Vissing-Jørgensen, C. (1993). "A finite step updating method for elastoplastic analysis of frames." Journal of Engineering Mechanics, 119, 2478-2495.

Krenk, S., Vissing-Jørgensen, C., and Thesbjerg, L. (1999). "Efficient collapse analysis techniques for framed structures." Computers \& Structures, 72, 481-496.

Liew, J. Y. R., Chen, H., Shanmugam, N. E., and Chen, W. F. (2000). "Improved nonlinear plastic hinge analysis of space frame structures." Engineering Structures, 22, 1324-1338.

Liew, J. Y. R., White, D. W., and Chen, W. F. (1993). "Second-order refined plastic-hinge analysis for frame design. Part I." Journal of Structural Engineering, 119, 3196-3217.

Lignos, D. G. and Krawinkler, H. (2011). "Deterioration modeling of steel components in support of collapse prediction of steel moment frames under eearthquake loading." Journal of Structural Engineering, ASCE, 137, 1291-1302.

Liu, Y., Xu, L., and Grierson, D. E. (2009). "Combined MVP failure criterion for steel cross-sections." Journal of Constructional Steel Research, 65, 116-124.

Lu, Y., Vintzileou, E., Zhang, G.-F., and Tassios, T. P. (1999). "Reinforced concrete scaled columns under cyclic actions." Soil Dynamics and Earthquake Engineering, 18, 151-167.

Mamaghani, I. H. P. and Kajikawa, Y. (1998). "Cyclic inelastic behavior of compact steel tubular columns." Tubular Structures, 8, 381-389.

Masi, A., Santarsiero, G., Lignola, G. P., and Verderame, G. M. (2013). "Study of the seismic behavior of external RC beam-column joints through experimental tests and numerical simulations." Engineering Structures, 52, 207-219.

Oran, C. (1973). "Tangent stiffness in space frames." Journal of the Structural Division, ASCE., 99, 987-1001.

Orbison, J. G., McGuire, W., and Abel, J. F. (1982). "Yield surface applications in nonlinear steel frame analysis." Computer Methods in Applied Mechanics and Engineering, $33,557-573$.

Popov, E. P., Mahin, S. A., and Zayas, V. A. (1980). "Inelastic cyclic behavior of tubular braced frames." Journal of the Structural Division, ASCE., 106, 2375-2390.

Powell, G. H. and Chen, P. F.-S. (1986). "3D beam-column element with generalized plastic hinges." Journal of Engineering Mechanics, ASCE, 112, 627-641.

Skordeli, M.-A. A. and Bisbos, C. D. (2010). "Limit and shakedown analysis of 3D steel 
frames via approximate ellipsoidal yield surfaces." Engineering Structures, 32, 15561567.

Ueda, Y., Akamatsu, T., and Ohmi, Y. (1969). "Elastic-plastic analysis of framed structures using the matrix method (in japanese)." Journal of the Society of Naval Architects of Japan, 126, 253-262.

Ueda, Y., Matsuishi, M., Yamakawa, T., and Akamatsu, Y. (1968). "Elastic-plastic analysis of framed structures using the matrix method (in japanese)." Journal of the Society of Naval Architects of Japan, 124, 183-191.

Ueda, Y. and Yao, T. (1982). "The plastic node method: A new method of plastic analysis." Computer Methods in Applied Mechanics and Engineering, 34, 1089-1104.

Willam, K. J. and Warnke, E. P. (1974). "Constitutive model for the triaxial behaviour of concrete." Proceedings of International Association of Bridge and Structural Engineering, 19, Sec. III, 1-30.

Zayas, V. A., Mahin, S. A., and Popov, E. P. (1980). "Cyclic inelastic behavior of steel offshore structures." Report No. Report UCB/EERC-80/27, EERC, Earthquake Engineering Research Center, Berkeley, CA. 